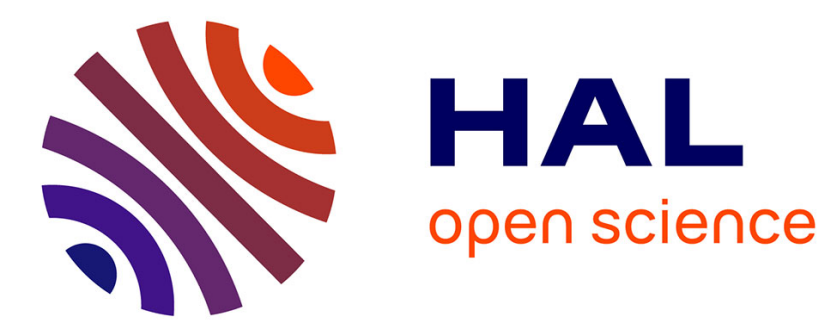

\title{
On-the-fly dynamics simulations of transient anions
}

\author{
F. Kossoski, M. T. Do N. Varella, M. Barbatti
}

\section{To cite this version:}

F. Kossoski, M. T. Do N. Varella, M. Barbatti. On-the-fly dynamics simulations of transient anions. Journal of Chemical Physics, 2019, 151 (22), pp.224104. 10.1063/1.5130547 . hal-02612332

\section{HAL Id: hal-02612332 \\ https://hal-amu.archives-ouvertes.fr/hal-02612332}

Submitted on 19 May 2020

HAL is a multi-disciplinary open access archive for the deposit and dissemination of scientific research documents, whether they are published or not. The documents may come from teaching and research institutions in France or abroad, or from public or private research centers.
L'archive ouverte pluridisciplinaire $\mathbf{H A L}$, est destinée au dépôt et à la diffusion de documents scientifiques de niveau recherche, publiés ou non, émanant des établissements d'enseignement et de recherche français ou étrangers, des laboratoires publics ou privés. 


\title{
On-the-Fly Dynamics Simulations of Transient Anions
}

\author{
F. Kossoski, ${ }^{* \dagger}$ M. T. do N. Varella, ${ }^{*, \ddagger}$ and M. Barbatti, ${ }^{*} \dagger$ \\ $\dagger$ †ix Marseille Univ, CNRS, ICR, Marseille, France \\ $\ddagger$ Institute of Physics, Universidade de São Paulo, Rua do Matão 1731, 05508-090 São \\ Paulo, Brazil
}

E-mail: fabris.kossoski@univ-amu.fr; mvarella@if.usp.br; mario.barbatti@univ-amu.fr

\begin{abstract}
A novel theoretical framework for describing the dynamics of transient anions is presented. An ensemble of classical trajectories is propagated on-the-fly, where resonance energies are computed with bound state techniques, and resonance widths are modeled with a combination of bound state and scattering calculations. The methodology was benchmarked against quantum dynamics results for model potential energy curves, and excellent agreement was attained. As a first application, we considered the electron induced dissociation of chloroethane. We found that electron attachment readily stretches the $\mathrm{C}-\mathrm{Cl}$ bond, which stabilizes the transient anion within $\sim 10$ fs and leads to the release of fast chloride ions. Both magnitude and shape of the computed dissociative electron attachment cross sections are very similar to the available experimental data, even though we found the results to be very sensitive on the accuracy of the underlying methods. These encouraging results place the proposed methodology as a promising approach for studies on transient anions dynamics of medium sized molecules.
\end{abstract}




\section{Introduction}

Among electron induced processes in molecular systems, dissociative electron attachment (DEA) plays a prominent role in a series of physico-chemical environments. Basically, a molecule captures an incoming low-energy electron $(\leq 10 \mathrm{eV})$ and forms a transient anion state, or resonance, which triggers off a nuclear dynamics that may culminate in dissociation. In interstellar space and planetary atmospheres, DEA may generate small inorganic and even prebiotic molecules. ${ }^{1}$ Cold plasmas, which are currently employed in many technological applications, ${ }^{2}$ rely on DEA of alkanes and haloalkanes in order to create species responsible for the devised treatment. One proposed application where DEA would be an active participant is the pre-treatment of lignocellulosic material, also based on cold plasmas. ${ }^{3}$ DEA is also recognized to have a range of implications in materials ${ }^{4,5}$ and environmental sciences. ${ }^{6,7} \mathrm{~A}$ major motivation concerns the interaction of low-energy electrons with biomolecules. ${ }^{8}$ They are very efficient in inducing single and double strand breaks in DNA, ${ }^{9}$ which may account for long-term biological damage. On the other hand, radiosentizer compounds typically present much larger DEA cross sections when compared to non-sensitizers, ${ }^{10,11}$ which suggest that electrons might play a decisive role in its bioactivity. DEA has also been suggested to participate in other biological processes. ${ }^{12,13}$ These and other situations where molecules fragment upon collision of low-energy electrons are reviewed in reference. ${ }^{4}$

In spite of its significance as a fundamental physico-chemical process, and its relevance to several fields, theoretical attempts to describe DEA are still limited to very small or to simplified systems. Non-local resonance theory, ${ }^{14}$ which is the most accurate treatment for electron induced processes, has been successfully employed for a series of diatomics or pseudo-diatomic models. ${ }^{15,16}$ High accuracy comes with high cost, and for larger molecules one usually employs a local approximation. ${ }^{14}$ Non-local effects are important for broad resonances or near-threshold phenomena. Otherwise, the local approximation provides similar results with a significantly reduced computational cost. ${ }^{14}$ Within the local approximation, the available theoretical contributions to the problem usually rely on building potential en- 
ergy surfaces with time consuming scattering calculations followed by nuclear wavepacket propagations. ${ }^{17-19}$ When considering all vibrational degrees of freedom, this approach is still restricted to diatomics and triatomics, ${ }^{18-20}$ and even then calculations are very costly. If the system of interest is a large molecule, one has to restrict the multidimensional potential energy surfaces to one ${ }^{21}$ or two ${ }^{17}$ vibrational coordinates. Molecular dynamics simulations are sometimes employed for the later stages of DEA, ${ }^{22-24}$ but without accounting for the autodetachment probability of its initial stage, when the anion is still transient. Alternatively, potential energy curves computed along reaction coordinates can also help understanding DEA mechanisms. ${ }^{25,26}$ Although theory has been providing significant contribution to the understanding of electron induced processes in small molecules, the current approaches are too computationally demanding to be applied to more complex systems and/or to more vibrational degrees of freedom. In view of these difficulties, the large amount of DEA data obtained in the last decades, from small ${ }^{27}$ to considerably complex biomolecules, ${ }^{12}$ remain mostly untouched by theory.

Faced with the various motivations related to DEA of molecular systems, the existence of abundant experiments on the process, and the limited contribution from theory to the problem up to date, we propose a new theoretical approach to investigate electron induced processes, based on mixed quantum-classical nuclear dynamics of the transient anion states. While this class of methods has been widely employed to survey species with constant charge state, it has not yet been adapted to describe transient anions, mostly due to the difficulty in describing autodetachment from these states.

In order to validate the proposed methodology, we have benchmarked it against quantum dynamics results for a series of model one-dimensional potential energy curves (PECs) for a single dissociative state. Then, we applied it to model DEA to an actual molecule. The chosen target should have a single low-lying shape resonance, count with available experimental DEA cross sections for comparison purposes, and should be larger than a diatomic or triatomic. Chloroethane $\left(\mathrm{CH}_{3} \mathrm{CH}_{2} \mathrm{Cl}\right)$ fulfills these requirements and was thus selected 
as the first case study. Electron attachment gives rise to a $\sigma^{*}$ resonance, which is centered at impact energies around $2.5 \mathrm{eV}$, as revealed by electron transmission spectroscopy (ETS) measurements ${ }^{28}$ and by scattering calculations. ${ }^{29}$ This resonance induces the elimination of the chloride ion $\left(\mathrm{Cl}^{-}\right)$, which has a maximum yield around $1.5 \mathrm{eV} .{ }^{30,31}$ Short lived resonances,

such as those of chloroalkanes, ${ }^{31-34}$ promote a shift from the vertical resonance energy to the maximum of the DEA cross section. This redshift is understood only at a qualitative level, and is related to the considerable difference in autodetachment lifetimes at energies lying below and above the center of the resonance. Our goals here are twofold. First, to propose the methodology and validate it by comparing to quantum dynamics results. And second, to demonstrate its potential and feasibility for a quantitative description of DEA to polyatomic molecules, by considering the chloroethane molecule as a test case.

\section{Theory}

The basic idea of our proposed computational strategy is to classically propagate nuclei on potential energy surfaces computed on-the-fly with bound state methods, while the probability of electron autodetachment is evaluated with an ad-hoc model built from electron scattering calculations. The first component of the problem comprises the nuclear dynamics, which we have described with mixed quantum-classical trajectory method. ${ }^{35-37}$ Basically, the full quantum mechanical nuclear wavepacket is replaced by an ensemble of independent and classical trajectories propagated on-the-fly, along adiabatic PECs that are computed as needed. It is thus expected that the ensemble average of an observable should behave similarly to its quantum mechanical expectation value. For many years now, this method has been successfully employed in photophysics and photochemistry problems, ${ }^{36,37}$ and we thus believe its extension to electron-induced processes should be equally reasonable. Next is the electronic description. Since the resonant anion is coupled to the continuum of scattering states, its description would ideally require the use of scattering methods ${ }^{38-40}$ or 
adapted bound state methods, such as the complex absorbing potential ${ }^{41,42}$ and the stabilization method. ${ }^{43,44}$ Conventional bound state methods can also be employed, ${ }^{45-47}$ as long as one ensures that the roots of the Hamiltonian correspond to the quasi-bound state (a valence anion state), rather than a pseudo-continuum state (the neutral molecule plus a free electron). This so-called variational collapse of the wavefunction can be circumvented by avoiding very diffuse functions. More importantly for the present discussion, methods that ignore the discrete-continuum coupling cannot provide information on the autodetachment probabilities, which is key for a quantitative description of resonant anions dynamics.

The discrete-continuum coupling of the transient state is given by the nonlocal resonance width $\Gamma(\mathbf{q}, E)$, which depends on both the impact energy $E$ and the nuclear coordinates of the target q. Within local descriptions, the energy dependence is substituted by the resonance energy $E_{r}$, which defines a local resonance width $\Gamma_{L}(\mathbf{q})=\Gamma\left[\mathbf{q}, E_{r}(\mathbf{q})\right] .{ }^{48}$ As long as the resonance width is not very large and thus does not overlap the collision threshold, local approximations provide very similar DEA cross sections to the full nonlocal description. ${ }^{48}$ In this case, the transient anion state can be characterized by a complexed value resonance energy $\epsilon$ :

$$
\epsilon=E_{r}-\frac{i}{2} \Gamma_{L}
$$

where the real component part $E_{r}$ defines the energy of the transient anion relative to the neutral, which is called resonance energy or resonance position. The decay of the state by electron detachment is accounted for by the imaginary component $-i \Gamma_{L} / 2$, where $\Gamma_{L}$ is the local resonance width (which controls the autodetachment rate), while $1 / \Gamma_{L}$ (in atomic units) is the autodetachment lifetime. Here and throughout the text we are going to use atomic units.

The calculation of DEA cross sections based on a classical formalism has been presented before. ${ }^{49,50}$ Much of the following construction is based on these previous works. There are, however, three key differences from their derivation to ours. First, Lehr et $a l^{49,50}$ have assumed a classical distribution for the nuclear degrees of freedom, while we adopt the Wigner 
distribution associated with the nuclear wavepacket, which has been shown to be much more accurate in other applications. ${ }^{51-53}$

Second, while we also employed the local resonance width $\Gamma_{L}(\mathbf{q})$ for the dynamics propagation, we kept the energy dependent resonance width $\Gamma(\mathbf{q}, E)$ in the attachment probability. In a sense, this is equivalent to the semilocal approximation, ${ }^{14,54}$ which uses the local width for the wavepacket propagation and the nonlocal version for the entrance and exit amplitudes. We further assumed energy and coordinate dependence of the width function to be separable: $\Gamma(\mathbf{q}, E)=g(\mathbf{q})^{2} \gamma(E)$, as is typically done in semilocal descriptions of quantum dynamics. ${ }^{14,48,54}$ The $g(\mathbf{q})$ function is built in such a way that the nonlocal width $\Gamma(\mathbf{q}, E)$ coincides with the local width $\Gamma_{L}(\mathbf{q})$ at the resonance energy $\left(E=E_{r}(\mathbf{q})\right)$ :

$$
g(\mathbf{q})^{2}=\frac{\Gamma_{L}(\mathbf{q})}{\gamma\left(E_{r}(\mathbf{q})\right)}
$$

such that:

$$
\Gamma(\mathbf{q}, E)=\Gamma_{L}(\mathbf{q}) \frac{\gamma(E)}{\gamma\left(E_{r}(\mathbf{q})\right)}
$$

And third, in Ref. ${ }^{49,50}$ the DEA cross section curves were obtained by transforming from coordinate to resonance energy variables, since the relation between these was known in their one-dimensional PEC. This is clearly not the case in actual molecules, which ultimately motivates the idea of sampling initial conditions and numerically propagating the trajectories. Here, the DEA cross sections are obtained by assigning a broadening function to each trajectory, as is usually done in calculations of photoabsorption cross sections. ${ }^{55}$

We start by considering the neutral target is prepared in a state whose Wigner distribution is given by $W(\mathbf{x})$, where $\mathbf{x}=\left(\mathbf{q}_{0}, \mathbf{p}_{0}\right)$ denotes a nuclear phase space point with coordinates $\mathbf{q}_{0}$ and momenta $\mathbf{p}_{0}$. The Wigner distribution $W(\mathbf{x})$ represents the probability density function (PDF) for finding the nuclear variables at $\mathbf{x}$. Now, the rate for electron attachment is given by $\Gamma\left(\mathbf{q}_{0}, E\right)$, and we assume it takes place when the kinetic energy of the incoming electron $E$ matches the resonance energy $E_{r}\left(\mathbf{q}_{0}\right)$, which brings in a delta function 
$\delta\left[E-E_{r}\left(\mathbf{q}_{0}\right)\right]$. This implies a vertical electron capture, in the sense that the nuclear degrees of freedom $\mathbf{x}$ remain unaltered. The multiplication of these factors provide the probability for electron attachment $p_{\text {att }}(\mathbf{x}, E) d \mathbf{x}$ :

$$
p_{\text {att }}(\mathbf{x}, E) d \mathbf{x}=\Gamma\left(\mathbf{q}_{0}, E\right) \delta\left[E-E_{r}\left(\mathbf{q}_{0}\right)\right] W(\mathbf{x}) d \mathbf{x} .
$$

Once the transient anion is formed, the nuclear coordinates evolve in time $(\mathbf{q}(\mathbf{x}, t))$ according to Hamilton's equations of motion, where we explicitly kept the dependence on the initial condition $\mathbf{x}$. Due to the time reversal symmetry of electron capture, both attachment and detachment rates at $\mathbf{q}$ must be the same and equal to $\Gamma_{L}(\mathbf{q})$. Instead of working with the detachment probability, it is more convenient to consider the survival probability $P_{\text {surv }}(\mathbf{x}, t)$, which is the probability that electron autodetachment has not taken place until time $t$ for the initial condition $\mathbf{x}$. At each instant $t$, the survival probability is decreased by the detachment probability, which provides the following equation:

$$
P_{\text {surv }}(\mathbf{x}, t+d t)=P_{\text {surv }}(\mathbf{x}, t)\left[1-\Gamma_{L}[\mathbf{q}(\mathbf{x}, t)] d t\right] .
$$

And given that $P_{\text {surv }}(\mathbf{x}, t=0)=1$ (electron capture sets the clock), the survival probability can be computed in closed form: ${ }^{49,56}$

$$
P_{\text {surv }}(\mathbf{x}, t)=\exp \left[-\int_{0}^{t} \Gamma_{L}\left[\mathbf{q}\left(\mathbf{x}, t^{\prime}\right)\right] d t^{\prime}\right] .
$$

As long as the resonance width is different from zero, the survival probability will be a monotonically decreasing function of time. When the anion becomes more stable than the neutral, autodetachment no longer takes place $\left(\Gamma_{L}=0\right)$, and $P_{\text {surv }}(\mathbf{x}, t)$ converges to the final survival probability, which we denote $P_{\text {surv }}(\mathbf{x})$. A scheme for the electron induced dynamics and the competition between DEA and autodetachment is represented in Fig. 1.

The mixed quantum-classical DEA cross section $\sigma$ is constructed by integrating the prod- 


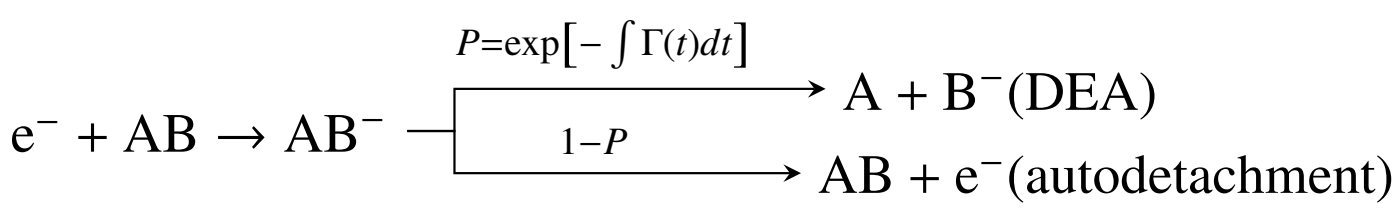
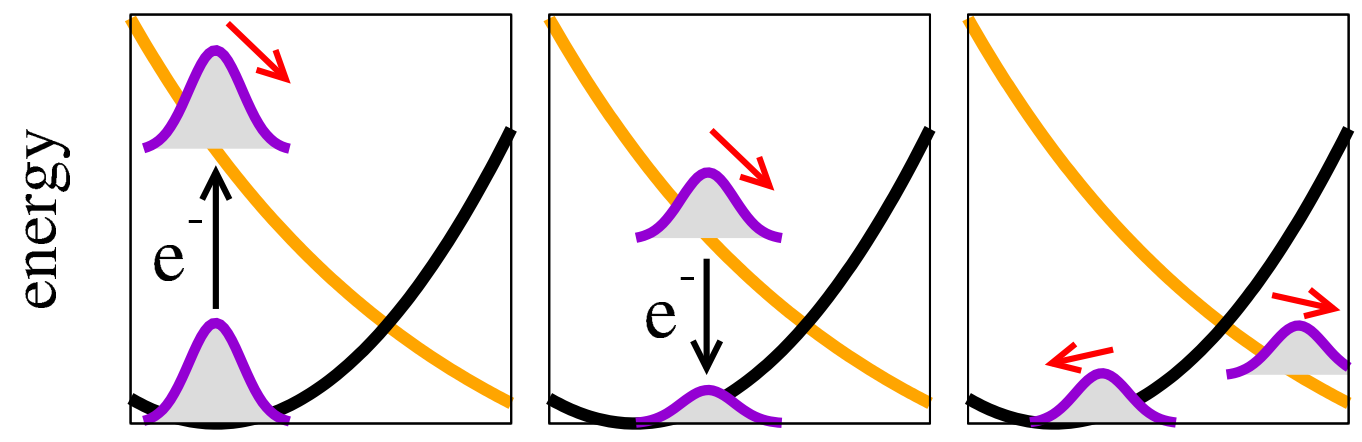

coordinate

Figure 1: Schematic representation of electron induced dynamics of the molecule AB. Initially (left panel), electron attachment promotes the nuclear wavepacket from the neutral ground state $\mathrm{AB}$ (black curve) to the transient anion state $\mathrm{AB}^{-}$(orange curve), thus triggering the dynamics of the nuclei (represented by the red arrow) along a dissociative coordinate. While the anion lies higher in energy than the neutral, the resonance width $\Gamma$ accounts for the possibility of electron detachment (middle panel). At the end (right panel), part of the wavepacket remains in the anion potential energy curve (accounting for dissociation), and part will lie in the neutral curve (accounting for vibrational excitation). The competition between dissociative electron attachment (DEA) and autodetachment is governed by the survival probability $P=\exp \left[-\int \Gamma(t) d t\right]$.

uct of the electron attachment $p_{\text {att }}(\mathbf{x}, E)$ and the survival $P_{\text {surv }}(\mathbf{x})$ probabilities over the initial conditions phase space $\mathbf{x}{ }^{49,57}$

$$
\sigma(E)=\frac{\pi}{E} \int \Gamma\left(\mathbf{q}_{0}, E\right) \delta\left[E-E_{r}\left(\mathbf{q}_{0}\right)\right] P_{\text {surv }}(\mathbf{x}) W(\mathbf{x}) d \mathbf{x}
$$

The integral in eq 7 is fit to be evaluated within the nuclear ensemble method. ${ }^{55}$ An ensemble of initial conditions is sampled according to the Wigner distribution $W(\mathbf{x})$, which effectively transforms the integral into an average over the ensemble. Given the constraint imposed by the delta function in eq 7 , each initial condition determines the energy $E$ for which the cross section is computed. Therefore, the energy dependence of the cross section is accounted for by the different initial resonance energies, which in turn reflects the underlying 
Wigner distribution. In order to shift from a collection of delta functions to an actual cross section curve, we have assigned a broadening function $g_{l}(\mathbf{x}, E)$ to each trajectory, similarly to what is done in photoabsorption cross section calculations. ${ }^{55}$ This also allows for the ratio $\gamma(E) / \gamma\left(E_{r}(\mathbf{q})\right)$ (eq 3) to become different from one when the impact energy does not coincide with the resonance energy. Here we have employed a generalized Breit-Wigner profile:

$$
g_{l}\left(\mathbf{q}_{0}, E\right)=\frac{N\left(\mathbf{q}_{0}\right)}{\pi} \frac{\eta\left(\mathbf{q}_{0}, E\right) / 2}{\left[E-E_{r}\left(\mathbf{q}_{0}\right)\right]^{2}+\left[\eta\left(\mathbf{q}_{0}, E\right) / 2\right]^{2}},
$$

where $N\left(\mathbf{q}_{0}\right)$ is a normalization constant ensuring that $\int_{0}^{\infty} g_{l}\left(\mathbf{q}_{0}, E\right) d E=1$. In particular, we have $g_{l}\left(\mathbf{q}_{0}, E\right) \sim \delta\left[E-E_{r}\left(\mathbf{q}_{0}\right)\right]$ when $\eta \rightarrow 0$. We could have used a constant broadening parameter $\left(\eta\left(\mathbf{q}_{0}, E\right)=\eta\right)$, which would give a Lorentzian function. Instead, we have incorporated the same $\gamma(E)$ function from the nonlocal width (eq 3) into the broadening function, which then leaves us with a single broadening parameter $\eta$ :

$$
\eta\left(\mathbf{q}_{0}, E\right)=\eta \frac{\gamma(E)}{\gamma\left(E_{r}\left(\mathbf{q}_{0}\right)\right)}
$$

Whether we adopt a constant or energy dependent broadening function makes a very small difference on the DEA cross section curves we have computed, but the later option should be more accurate for resonances closer to $0 \mathrm{eV}$, as Wigner's threshold law ${ }^{58}$ would be accounted for with an appropriate $\gamma(E)$.

When accounting for the separable width function, the initial conditions sampling and the broadening function, the working expression for the DEA cross sections becomes:

$$
\sigma(E)=\frac{\pi}{E} \frac{1}{N} \sum_{i=1}^{N} \frac{\gamma(E)}{\gamma\left(E_{r}\left(\mathbf{q}_{0}^{i}\right)\right)} \Gamma_{L}\left(\mathbf{q}_{0}^{i}\right) g_{l}\left(\mathbf{q}_{0}^{i}, E\right) P_{\text {surv }}\left(\mathbf{x}^{i}\right)
$$

where the superscript $i$ denotes one of the $N$ trajectories, whose initial conditions $\mathbf{x}^{i}=$ $\left(\mathbf{q}_{0}^{i}, \mathbf{p}_{0}^{i}\right)$ are sampled from the Wigner distribution.

For the dynamics of chloroethane, the autodetachment lifetime was modeled as follows. 
First, we have assumed that the local resonance width dependence on position can be approximated by an explicit function of the corresponding resonance energy:

$$
\Gamma_{L}[\mathbf{q}(\mathbf{x}, t)] \simeq \Gamma_{L}\left[E_{r}(\mathbf{q}(\mathbf{x}, t))\right]
$$

We performed ab-initio electron scattering calculations (to be described in Sec. 3.5), for four geometries where the $\mathrm{C}-\mathrm{Cl}$ bond distance was varied, which provided pairs of resonance energies and widths. We further included the point $\left(E_{r}=0, \Gamma_{L}=0\right)$ into the set, which guarantees that autodetachment cannot take place when the anion becomes electronically stable. Since we found resonance energies and widths to be quite correlated, the instantaneous $\Gamma_{L}\left[E_{r}(\mathbf{q}(\mathbf{x}, t))\right]$ was obtained from a third-order polynomial interpolation ${ }^{59}$ to the computed points. Accounting only for the $\mathrm{C}-\mathrm{Cl}$ stretching coordinate when building our model for the resonance width is justified a posteriori, since the dynamics simulation reveals that the anion relaxes mainly via the elongation of the $\mathrm{C}-\mathrm{Cl}$ bond. More sophisticated models (that include an explicit dependence on the vibrations) could be devised, but that is beyond the scope of the present work. But based on the character of the state and on the actual results from the dynamics, we anticipate the effect of the other degrees of freedom to be of lesser importance to the resonance width.

While the scattering calculations served mainly to provide a reliable model for the autodetachment probabilities, they were not employed for the calculation of energies and gradients during the dynamics. Rather, bound state methods (to be described in Sec. 3.4) were employed for the calculation of anion energies $\left(V_{d}\right)$ and energy gradients, as well as for a single point calculation of the neutral ground state energy $\left(V_{0}\right)$, which then provided the instantaneous resonance energy $\left(E_{r}\right)$. We stress that a key benefit of the proposed methodology lies on the observation that resonance positions can be estimated based on bound state methods, and these are usually less computationally demanding than scattering calculations. Furthermore, the former class of methods usually offers analytical nuclear gradients (pivotal 
for the efficiency of the dynamics), which is not the case for the later. Finally, the computed resonance energy is employed in the above-described autodetachment model in order to evaluate the local resonance width $\left(\Gamma_{L}\right)$ at each timestep. It is also worth mentioning that the dynamics propagation and the autodetachment model are two independent aspects of the problem. Therefore, improved autodetachment models could be devised, without the need to repeat the computationally demanding dynamics simulations. Moreover, although we have not investigated this aspect here, this methodology can be directly connected to surface hopping trajectories. ${ }^{60}$

\section{Computational details}

\subsection{Quantum dynamics}

For the quantum propagation in the model PECs, we have employed the semi-local version

of the Feshbach projection operator formalism. ${ }^{48}$ Since the employed models involve one nuclear degree of freedom, we will use the dissociative coordinate $R$ instead of $\mathbf{q}$. In the semi-local approximation, the entrance amplitudes incorporate the non-local $\Gamma(R, E)$ width, while the local $\Gamma_{L}(R)$ width is employed for the wavepacket propagation: ${ }^{48}$

$$
i \frac{\partial}{\partial t} \tilde{\psi}_{d}(R, t)=\left[T_{n}+V_{d}(R)-\frac{i}{2} \Gamma_{L}(R)\right] \tilde{\psi}_{d}(R, t),
$$

whose initial condition is given by:

$$
\tilde{\psi}_{d}(R, 0)=g(R) \chi_{\nu}(R) .
$$

In the above equations, $T_{n}=-(2 \mu)^{-1} d^{2} / d R^{2}$ is the nuclear kinetic energy operator, $\mu$ is the reduced mass, $V_{d}(R)$ and $\Gamma_{L}(R)$ are respectively the real and imaginary components of the anion PEC, $g(R)$ is related to $\Gamma_{L}(R)$ via eq 2 , and $\chi_{\nu}(R)$ is the initial vibrational state. 
Once the wavepacket is propagated, the DEA cross section can be computed as: ${ }^{48}$

$$
\sigma(E)=\frac{\pi}{2 E} \frac{K}{\mu} \gamma(E)\left|\lim _{R \rightarrow \infty} e^{-i K R} \int_{0}^{\infty} d t \tilde{\psi}_{d}(R, t) e^{i E t}\right|^{2}
$$

where $K=\sqrt{2\left(E-E_{\mathrm{thr}}\right)}$ is the relative momentum of the departing fragments and $E_{\mathrm{thr}}$ is the energy threshold for the DEA reaction.

The numerical propagation was performed with the split-operator technique. ${ }^{61}$ The wavepacket is multiplied by the (real and imaginary) potential energy in position representation and by the kinetic energy in momentum representation, while the transformation between representations is performed with fast Fourier transform. ${ }^{62}$ We employed a space grid of $2^{10}=1,024$ points, from $-2.0 a_{0}$ to $6.0 a_{0}$, and a temporal grid of $2^{18}=262,144$ points, from 0 fs to 500 fs (which corresponds to a timestep of $3.8 \times 10^{-3} \mathrm{fs}$ ). In order to avoid spurious reflexions of the wavepacket from the end of the grid, the amplitude of the wavepacket lying beyond the cutoff radius $R_{\text {cut }}$ was damped by multiplying it by the term $\cos \left[(\pi / 2)\left(R-R_{\text {cut }}\right) /\left(R_{\text {last }}-R_{\text {cut }}\right)\right]{ }^{48}$ where $R_{\text {last }}$ is the last grid point. Finally, the limit in eq 14 is taken at a finite $R=R_{\text {DEA }}$ position, beyond the crossing point of anion and neutral PECs, and before $R_{\text {cut }}$. Here we have used $R_{\mathrm{DEA}}=4.0 a_{0}$ and $R_{\text {cut }}=4.1 a_{0}$.

\subsection{Distorted sampling distribution}

As already pointed out, the ensemble of initial conditions for the dynamics simulations should be sampled according to the appropriate Wigner distribution. Here we consider each normal mode to be given by a harmonic oscillator potential, whose normalized Wigner distribution for finite temperatures ${ }^{63,64}$ is given by:

$$
W(\mathbf{q}, \mathbf{p})=\prod_{i=1}^{M}\left(\frac{\alpha_{i}}{\pi}\right) \exp \left(-\frac{q_{i}^{2}}{2 \sigma_{q i}^{2}}-\frac{p_{i}^{2}}{2 \sigma_{p i}^{2}}\right)
$$


with $\sigma_{q i}^{2}=1 /\left(2 \alpha_{i} \mu_{i} \omega_{i}\right), \sigma_{p i}^{2}=\mu_{i} \omega_{i} /\left(2 \alpha_{i}\right), \alpha_{i}=\tanh \left(\omega_{i} / 2 T\right), \omega_{i}$ and $\mu_{i}$ are the vibrational frequency and reduced mass of mode $i$, and $T$ is the temperature.

Alternatively, we could sample from any other sampling PDF, and employ the importance sampling technique to map the results to the target PDF of interest, as has been recently demonstrated. ${ }^{65}$ Basically, each trajectory is assigned an importance sampling weight (the ratio between target and sampling PDF evaluated at the sampled point), which exactly compensates for the difference between the two PDFs. There are two situations in which one would employ different sampling and target PDFs. First, when a set of calculations has already been performed for one sampling PDF, the results can be mapped into any other target PDF. This is the case discussed in Ref, ${ }^{65}$ where the importance sampling technique was employed to compute temperature dependent observables. The second motivation is related to a situation when sampling from the target PDF leads to a poor convergence of the observable being computed. In this case, one should adopt a PDF that better samples from the most important region of the parameter space. While this does not seem to be a reason for concerns in simulations of photoexcited states, it proved to be a major issue in the present dynamics of short-lived transient anions, as will be discussed in Sec. 4. Here we have performed importance sampling calculations for both situations discussed above.

The implementation of the importance sampling technique for the selection of initial conditions ${ }^{65}$ was generalized by allowing for arbitrary broadened and displaced Gaussians as PDFs. Denoting $\mathbf{x}=(\mathbf{q}, \mathbf{p})$ as the phase space point with coordinate $\mathbf{q}$ and momentum $\mathbf{p}$, a Gaussian distribution centered at $\mathbf{x}_{0}=\left(\mathbf{q}_{0}, \mathbf{p}_{0}\right)$ with standard deviation $\sigma=\left(\sigma_{q}, \sigma_{p}\right)$ is given by:

$$
P(\mathbf{x})=\prod_{i=1}^{2 M} \frac{1}{\sqrt{2 \pi} \sigma_{i}^{2}} \exp \left(-\frac{\left(x_{i}-x_{i 0}\right)^{2}}{2 \sigma_{i}^{2}}\right)
$$

In the most general case, displacements and standard deviations for both sampling and target distribution could be different, and the importance sampling weights would be calculated by the ratio of the corresponding distributions, as given by eq 16 . When distributions are displaced from one another, but share the same Gaussian exponent $\sigma$, then the impor- 
tance sampling weights are computed as:

$$
w(\mathbf{x})=\prod_{i=1}^{2 M} \frac{\sigma_{i}^{s}}{\sigma_{i}^{t}} \exp \left(-\frac{\left(x_{i 0}^{t}\right)^{2}-\left(x_{i 0}^{s}\right)^{2}}{2 \sigma_{i}^{2}}-\frac{x_{i 0}^{t}-x_{i 0}^{s}}{\sigma_{i}^{2}} x_{i}\right)
$$

where the superscript $s$ and $t$ denote sampling and target PDF parameters.

\subsection{Classical dynamics}

100,000 trajectories were propagated for each of the model PECs. The dynamics simulations of the chloroethane resonant anion were performed with the Newton-X package ${ }^{66,67}$ interfaced with the Columbus ${ }^{68,69}$ software. The electronic problem was described at the multireference configuration interaction (MRCI) level, ${ }^{70}$ with two levels for the excitation number: zero (MRCI $(0)$ ), and two (MRCI with singles and doubles, or MRCISD), which will be described in Sec 3.4. 1,400 trajectories were run for the MRCISD calculations, while the dynamics performed at the $\mathrm{MRCI}(0)$ level counted with 1,000 trajectories. Nuclei were propagated numerically according to Velocity-Verlet algorithm, with a timestep of 0.5 fs. This timestep did not compromise the calculation of the survival probability, as they were very close to those obtained by employing a timestep of $0.1 \mathrm{fs}$, for 10 selected initial conditions. Furthermore, due to the exponential dependence of the survival probability on the resonance width, its calculation could become inaccurate if we were to evaluate the integral in eq 6 with the same timestep of the dynamics. Instead, we have integrated a third-order polynomial interpolation ${ }^{59}$ of the $\Gamma_{L}[\mathbf{q}(t)]$ function, with a timestep of $0.002 \mathrm{fs}$, which guaranteed a smooth variation of the exponential argument. If one is interested in evaluating the initial relaxation of the transient anion and also in computing DEA cross sections, it is enough to propagate the dynamics until the anion becomes more stable than the neutral. Thus, whenever the $\mathrm{C}-\mathrm{Cl}$ distance reached $4.5 a_{0}$, the trajectory was terminated, which is more than enough to guarantee stabilization against autodetachment. On the other hand, if one is also interested in how the initially deposited energy is distributed between the fragments, 
than the dynamics should contemplate the drifting of the fragments until their interaction has considerably decreased. We have analyzed this later stage of DEA by proceeding with the propagation of 200 trajectories beyond the $4.5 a_{0}$ cutoff. The bound state calculations (MRCISD/MRCI(0)) eventually failed to converge, which typically happened for a $\mathrm{C}-\mathrm{Cl}$ distance of around $13 a_{0}$.

Initial conditions for the dynamics simulations of chloroethane were generated by independently sampling position and momentum for each normal mode. Except for the $\nu_{3}$ normal mode (which is dominated by the $\mathrm{C}-\mathrm{Cl}$ stretching), all other modes were sampled according to the harmonic potential Wigner distribution for a temperature of $333 \mathrm{~K}$ (eq 15), which matches the experimental condition of the DEA cross section measurements. ${ }^{31}$ As for the $\nu_{3}$ mode, we adopted a displaced Wigner distribution (eq 16) with $q_{\nu_{3}, 0}^{s}=1.5 /\left(\mu_{3} \omega_{3}\right)$ and $p_{\nu_{3}, 0}^{s}=1.0 \mu_{3} \omega_{3}$, both in the direction of promoting the dissociation. This bias showed to be critical in order to attain a reasonable convergence of the DEA cross sections with the number of trajectories. The centers of the sampling PDF were chosen after testing a couple of combinations for a very reduced set of trajectories. Finally, the bias on the sampling is corrected to the actual $333 \mathrm{~K}$ Wigner distribution by means of the importance sampling weights (eq 17).

\subsection{Bound state calculations}

Describing resonant states with bound state methods is expected to be a rather tricky task. On the one hand, anion states usually require more diffuse functions than neutral states. However, when very diffuse functions are employed, the additional electron of the anion state does not lie in a valence-type orbital, but rather in a diffuse orbitals far from the molecular frame. In this case, the anion state is actually emulating the scattering continuum and not the resonant anion, which has a valence nature. One can circumvent this issue by choosing a basis set that effectively constraint the electron to occupy a valence orbital. We found the aug-cc-pVDZ basis set ${ }^{71}$ offered a good compromise between both requirements. The 
exception would be at geometries where the $\mathrm{C}-\mathrm{Cl}$ bond is compressed, where the computed anion state mimics the continuum, but these geometries are unimportant for the dissociation and were never actually sampled for the dynamics. Instead of this simpler approach of employing an already described basis set, one should ideally search for an adequate set of basis functions with the aid of stabilization methods, ${ }^{25,72}$ but we did not pursue that here. Another major methodological challenge concerns a balanced description of correlation effects for neutral and anion states. In view of these inerent difficulties, our choice of basis set and electronic structure methods should imply in an overestimation of the resonance position. Employing more sophisticated techniques (such as the stabilization method and higher levels of electronic structure theory) should in principle provide more accurate resonance energies, but that would make our proposed on-the-fly dynamics methodoly impracticable. Here we have corrected the resonance energies computed with bound state methods in order to match the result obtained from ab-initio scattering calculations (to be described in Sec. 3.5) performed at the neutral equilibrium geometry.

The active orbitals of chloroethane were generated in a state-averaging (SA) complete active space self-consistent field (CASSCF) calculation, or SA-CASSCF, which included the ground state and two singlet excited states of the neutral in the SA. 6 electrons were distributed in 4 orbitals: the bonding $\sigma_{\mathrm{CCl}}$ and anti-bonding $\sigma_{\mathrm{CCl}}^{*}$ orbitals at the $\mathrm{C}-\mathrm{Cl}$ bond and the two non-bonding $n_{\mathrm{Cl}}$ orbitals at the chlorine atom. Augmenting this active space with orbitals located at the $\mathrm{C}-\mathrm{C}$ bond had a small effect $(\sim 0.1 \mathrm{eV})$ on the energies of the resonance and thus we kept with the $(6,4)$ active space. Performing CASSCF calculations for the anion state usually resulted in diffuse orbitals replacing the $\sigma^{*}$ orbital in the active space, as placing the extra electron far from the molecule provides a lower electronic energy than that of the valence $\sigma^{*}$ anion. In order to avoid the appearance of continuum intruder states, both anion and neutral states shared the same active orbitals as obtained from the above described SA(3)-CASSCF level. Attempts to include the anion state in the SA also led to the same issue. The electronic wavefunction was described at the MRCI level, ${ }^{70}$ with 
a $(6,4)$ reference space for the neutral and a $(7,4)$ space for the anion, and with both zero $(\mathrm{MRCI}(0))$ and two (MRCISD) excitations from the active space. For the neutral state the $\mathrm{MRCI}(0)$ energy is equivalent to the first root of the SA(3)-CASSCF calculation. On the other hand, the MRCI(0) calculation is not the same as a CASSCF calculation performed for the anion, since the orbitals of the former are optimized according to the SA(3)-CASSCF calculation. For the static calculations the Davidson correction to the MRCISD wavefunction $(\mathrm{MRCISD}+\mathrm{Q})^{73}$ was computed.

\subsection{Scattering calculations}

In order to provide a reliable model for the resonance lifetime, and also to validate the results obtained from the bound state calculations, we have performed fixed-nuclei elastic scattering calculations, at the equilibrium geometry obtained from the MRCISD level and also for a couple of distorted geometries. We employed the Schwinger multichannel method (SMC), ${ }^{40,74,75}$ with pseudopotentials, ${ }^{76}$ in its latest computational implementation. ${ }^{77}$ The same Cartesian Gaussian functions presented in Ref. ${ }^{78}$ (generated as in Ref. ${ }^{79}$ ) were employed here, while one p-type function with a 0.75 exponent was supplemented to the hydrogen atoms. An additional set of diffuse $4 \mathrm{~s} 3 \mathrm{p}$ functions was placed at the carbon atom bonded to the chlorine, with exponents generated by successive divisions by 4 , as proposed in Ref. ${ }^{80}$ The neutral ground state was described at the restricted Hartree-Fock level, making use of the Gamess package, ${ }^{81}$ while modified virtual orbitals ${ }^{82}$ were employed for the scattering calculations, as generated in the field of the cation with charge +4 . The use of MVOs is a standard procedure in scattering calculations. ${ }^{83}$ They should provide a more compact representation of correlation-polarization effects (thus requiring less CSFs), since they have a more valence-like character than the canonical virtual orbitals.

The scattering wavefunction was expanded in a set of configuration state functions (CSFs) $\left\{\left|\chi_{m}\right\rangle\right\}$, built as $\left|\chi_{j}\right\rangle=A\left[\left|\Phi_{0}\right\rangle \otimes\left|\varphi_{j}\right\rangle\right]$ (which accounts for static and exchange interactions), and $\left|\chi_{i j}\right\rangle=A\left[\left|\Phi_{i}\right\rangle \otimes\left|\varphi_{j}\right\rangle\right]$ (which incorporates polarization and correlation effects). For the 
former type of CSFs each virtual orbital (scattering orbital) $\left|\varphi_{j}\right\rangle$ is coupled to the target ground state $\left|\Phi_{0}\right\rangle$ and then antisymmetrized with the operator $A$. The later type was generated by coupling a scattering orbital $\left|\varphi_{j}\right\rangle$ to a single virtual excitations $\left|\Phi_{i}\right\rangle$ of the target (which define a pair of hole and particle orbitals). This set of functions were selected based on the criteria $\varepsilon_{\text {scat }}+\varepsilon_{\text {part }}-\varepsilon_{\text {hole }}<\varepsilon_{\text {cut }},{ }^{84}$ for an energy cutoff $\varepsilon_{c u t}=1.6$ Hartree, where the $\varepsilon$ 's are the energies of the scattering, particle and hole orbitals. Both singlet- and tripletcoupled target excitations were considered, while only the spin preserving doublet CSFs were kept in the scattering wavefunction expansion. Since we are interested in characterizing the $\sigma^{*}$ resonance, the scattering calculations were performed only for the $A^{\prime}$ irreducible representation. The three lowest singular vectors of the SMC denominator matrix were removed from the calculation, since they were assigned to linear dependency problems. The same protocol was adopted for the scattering calculations performed at nuclear configurations where the $\mathrm{C}-\mathrm{Cl}$ bond was stretched.

At each $\mathrm{C}-\mathrm{Cl}$ bond distance $R$, energy $E_{r}(R)$ and width $\Gamma_{L}(R)$ of the resonance were obtained from the scattering calculations by fitting the computed eigenphase sum $\delta(R, E)$ to a functional form comprising a Breit-Wigner profile and a second-order degree polynomial in energy:

$$
\delta(R, E)=-\tan ^{-1}\left(\frac{\Gamma_{L}(R) / 2}{E-E_{r}(R)}\right)+a_{0}+a_{1} E+a_{2} E^{2}
$$

Meanwhile, the $\gamma(E)$ function was obtained with a single fit to the equilibrium geometry eigenphase sum, according to: ${ }^{48}$

$$
\delta\left(R_{0}, E\right)=-\tan ^{-1}\left(\frac{\gamma(E) / 2}{E-\epsilon_{d}-\Delta(E)}\right)+a_{0}+a_{1} E^{1 / 2}+a_{2} E
$$

with

$$
\begin{aligned}
\gamma(E) & =A E^{1 / 2} \exp (-b E) \\
\Delta(E) & =\frac{A}{2}\left[-\frac{1}{\sqrt{\pi b}}+E^{1 / 2} e^{-b E}|\operatorname{erf}(i \sqrt{b E})|\right]
\end{aligned}
$$


where erf is the error function. According to the Wigner threshold law, at lower collision energies the scattered electron decays with the lowest partial wave allowed by the target symmetry. ${ }^{58}$ For polar molecules, the threshold behaviour is dominated by the s-wave $(l=0)$, such that $\gamma(E) \sim E^{1 / 2}$ for $E \rightarrow 0$. It is important to realize that the resonant contribution (as given via the $\tan ^{-1}$ functional form in Eqs. 18 and 19), and the threshold behavior (as governed by the $\gamma(E)$ function) are two separate aspects of the scattering eigenphase. In fact, while the $\sigma^{*}$ resonance of chloroethane is mostly of p-wave $(l=1)$ character at the local resonance energy, the s-wave prevails close to the threshold. The set of parameters obtained from the eigenphase sum fitting can be found in the Supporting Information.

At this point, we briefly review how each term of the working expression for the DEA cross sections is evaluated (see Eq. 10). The local resonance width $\Gamma_{L}\left(\mathbf{q}_{0}^{i}\right)$ is evaluated at each sampled initial condition $\mathbf{q}_{0}^{i}$, and its expression is built from a few scattering calculations, as explained after Eq. 11. The $\gamma(E)$ function is given by Eq. 21, and is obtained from a single scattering calculation performed at the neutral equilibrium geometry, as described above. In the denominator it is evaluated at the resonance energy of each initial condition $E_{r}\left(\mathbf{q}_{0}^{i}\right)$, while in the numerator it is evaluated in terms of the continuous electron impact energy $E$ variable. The broadening function $g_{l}\left(\mathbf{q}_{0}^{i}, E\right)$ is given by Eq. 8, and depends on the initial resonance energy $E_{r}\left(\mathbf{q}_{0}^{i}\right)$ and the $\gamma(E)$ function. And the survival probability $P_{\text {surv }}\left(\mathbf{x}^{i}\right)$ is computed according to Eq. 6, thus requiring the resonance width along the trajectory $\mathbf{q}\left(\mathbf{x}^{i}, t^{\prime}\right)$. Therefore, results from the actual propagation of the trajectories enter solely in the survival probability, while the others terms depend only on the initial condition $\mathbf{q}_{0}^{i}$. Finally, the cross section curve is obtained by multiplying each term and averaging over the ensemble of $N$ trajectories. 


\section{Model potential energy curves}

A harmonic potential was employed for the neutral PEC $V_{0}(R)$ and a single-exponential dissociative curve for the anion PECs $V_{d}(R)$, while the local resonance width $\Gamma_{L}(R)$ was modeled as being proportional to the resonance energy $E_{r}(R)=V_{d}(R)-V_{0}(R)$ :

$$
\begin{aligned}
V_{0}(R) & =C R^{2}, \\
V_{d}(R) & =\left[E_{r}^{0}-D\right] \exp (-\alpha R)+D, \\
\Gamma_{L}(R) & =\max \left[\frac{\Gamma^{0}}{E_{r}^{0}} E_{r}(R), 0\right] .
\end{aligned}
$$

The max function ensures that $\Gamma_{L}(R)=0$ whenever the anion is more stable than the neutral $\left(E_{r}(R)<0\right)$. In all models considered, we have fixed the neutral PEC $(C=1.5 \mathrm{eV})$, the vertical resonance energy $\left(E_{r}^{0}=2 \mathrm{eV}\right)$ and the asymptote of the anion $\operatorname{PEC}(D=-1 \mathrm{eV})$. Unless stated otherwise, the reduced mass was taken as $\mu=9$ a.m.u. The explicit energy dependent part of the resonance width was modeled as:

$$
\gamma(E)=A E^{l+1 / 2} \exp (-b E)
$$

with $A=1, l=1$ and $b=0.5 \mathrm{eV}^{-1}$.

We start by discussing the results for the reference model A1, where $\alpha=0.8 a_{0}^{-1}$ and $\Gamma^{0}=$ $1 \mathrm{eV}$. The PECs and both the actual Wigner distribution and a displaced $\left(q_{0}^{s}=0.2 /(\mu \omega)\right)$ Wigner distribution are presented in Figure 2. We have computed the energy integral of the DEA cross section for an increasing number of trajectories, when sampling from both shifted and unshifted Wigner PDFs. In the former case, we employed importance sampling weights (eq 17) to correct for the different sampling and target (unshifted Wigner distribution) PDFs. As can be seen in Figure 2, both approaches lead to the same result for a sufficiently large number of trajectories, as they should. Importantly, the convergence is dramatically improved when sampling from the shifted Wigner distribution. This happens because of the 

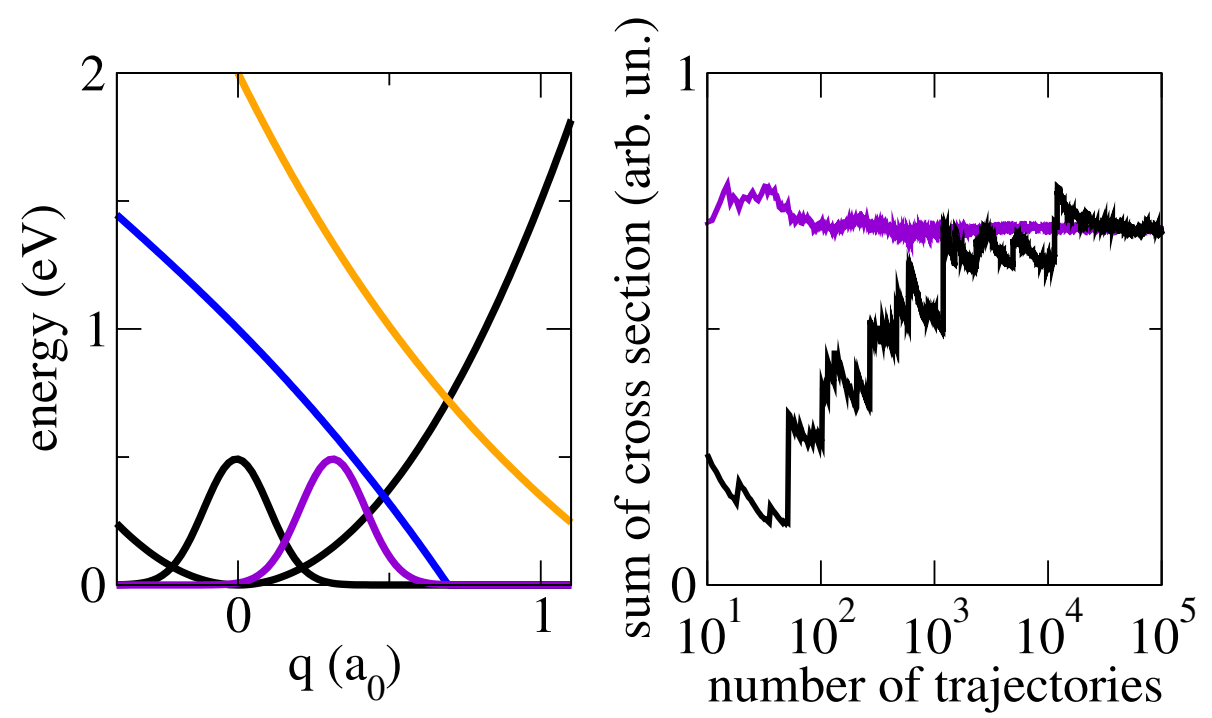

Figure 2: On the left, PECs for the A1 model: neutral state (black) and real (orange) and imaginary (blue) components of the anion state, together with the corresponding Wigner distribution of the neutral vibrational ground state (black) and a displaced distribution (purple). On the right, energy integral of the DEA cross section (in arbitrary units) for an increasing number of trajectories, computed for both original (black) and displaced (purple) Wigner distribution as the sampling distribution, with the former as the target distribution.

much larger survival probabilities obtained for trajectories starting with the more positive position and momentum. They start closer to the crossing point and reach it faster, such that autodetachment is less likely and takes place during a shorter period of time, thus decreasing the argument of the exponential in the survival probability (eq 6). On the other hand, sampling an initial condition that provides larger survival probabilities becomes increasingly unlikely, due to the Gaussian decay of the Wigner distribution. When accounting for the opposing trends of the survival probability and the initial condition sampling distribution, there should be an intermediate region in phase space which maximizes the likelihood of DEA. Therefore, when there is good overlap between this important phase space region and the sampling PDF, the convergence of the DEA cross section should improve, as we have observed. For all model PECs, we employed broadening and shifting parameters for the sampling PDF that provided a similar convergence as that obtained for the A1 model. One could in principle optimize the sampling PDF in order to maximize the rate of convergence, but we did not pursue that. When dealing with dynamics simulations of photoexcited 
molecules, one can usually afford to run on the order of few hundred trajectories based on ab initio methods to few thousand trajectories based on semiempirical methods. For transient anions which present a sizable shift from the vertical resonance energy to the maximum of the DEA cross section, as is the case of chloroethane, converged observables would only be attained with the aid of the importance sampling technique ${ }^{65}$ and a well suited sampling PDF.

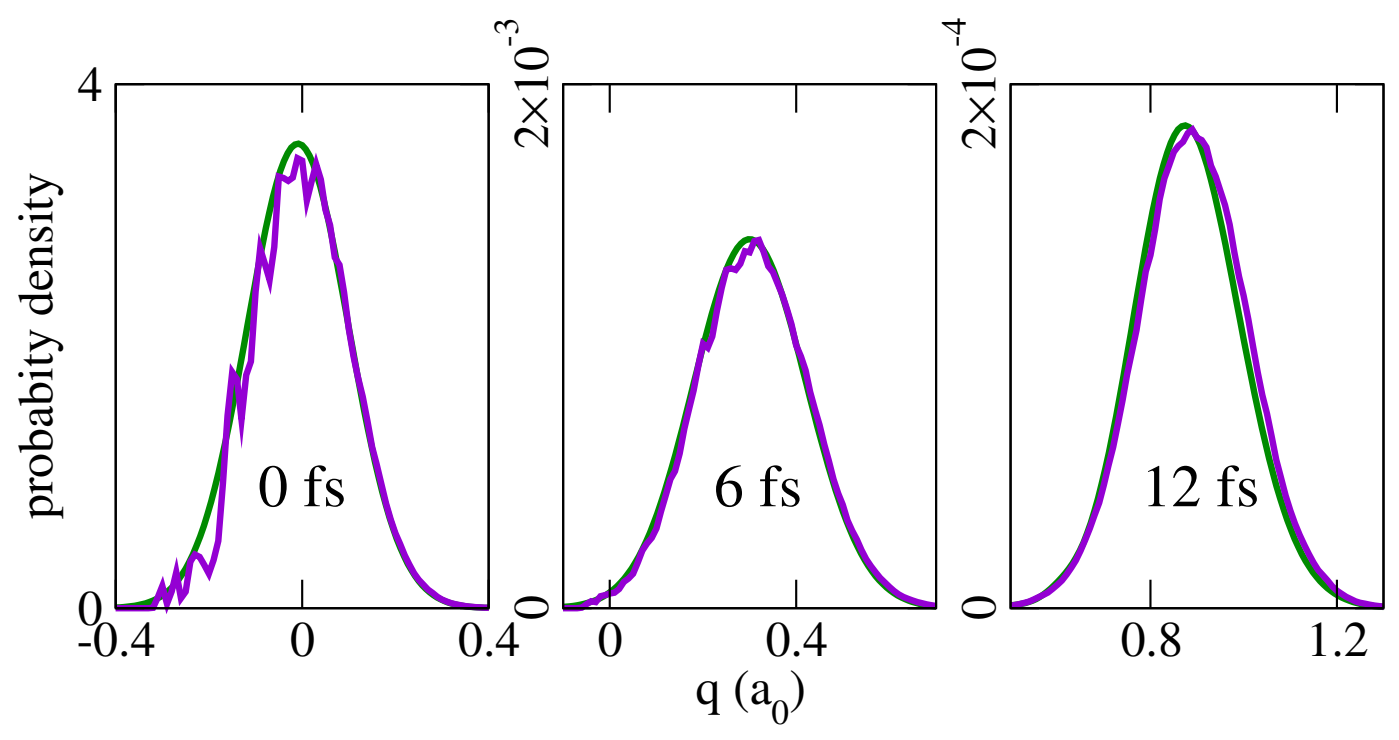

Figure 3: Quantum wavepacket (green) and distribution of classical trajectories (violet) for the A1 model, at $t=0 \mathrm{fs}, t=6 \mathrm{fs}$ and $t=12 \mathrm{fs}$.

The wavepacket (as obtained from the quantum propagation) and the distribution function for the classical trajectories are depicted in Figure 3, for propagation times of $t=0$ fs, $t=6 \mathrm{fs}$ and $t=12 \mathrm{fs}$. The classical distribution was obtained by attaching a narrow Gaussian function to each trajectory and summing over the ensemble. The matching between the curves is quite remarkable. Not only the classical propagation correctly mimics the displacement and speed of the wavepacket, but it also reproduces the four order of magnitude decrease in its norm. This last point demonstrates that assigning a survival probability (eq 6) as an effective weight to each trajectory provides an excellent approximation to the action of the imaginary component operator $-i \Gamma_{L}(\mathbf{q}) / 2$ on the quantum wavepacket.

Besides the reference model A1, we have tested for other combinations of the parameters 
$\alpha$ and $\Gamma^{0}$. The former provides the steepness of the PEC, and is thus the key parameter controlling the dissociation lifetime, while the later governs the magnitude of the resonance width, hence the autodetachment lifetimes. The following values were considered: $\alpha=0.8 a_{0}^{-1}$ (model A), $\alpha=0.5 a_{0}^{-1}$ (model B) and $\alpha=0.2 a_{0}^{-1}$ (model $\left.\mathrm{C}\right)$, and $\Gamma^{0}=1 \mathrm{eV}(\operatorname{model} 1)$, $\Gamma^{0}=0.2 \mathrm{eV}$ (model 2 ) and $\Gamma^{0}=0.04 \mathrm{eV}$ (model 3$)$. Each pair of parameters was combined, which gave rise to nine sets of model PECs. For example, $\alpha=0.8 a_{0}^{-1}$ and $\Gamma^{0}=1 \mathrm{eV}$ defines the model A1, and so on. Even though these parameters were arbitrarily chosen, they should be representative of what one could encounter in actual molecules. Figure 4 shows the nine model PECs considered, while the corresponding DEA cross sections computed according to both quantum (eq 14) and mixed quantum-classical (eq 7) propagations are presented in Figure 5. Overall, agreement is excellent. The main difference lies on a small shift of the mixed quantum-classical DEA cross section peak position to lower energies. This shift virtually disappears when the resonance width is decreased (moving from left to right panels in Figures 4 and 5), but becomes more pronounced when the anion PEC becomes less steep (moving from top to bottom panels). To a great extent, the redshift in the DEA cross sections can be traced back to the small differences between the quantum wavepacket and the ensemble of classical trajectories. In the A1 model, we found the development of a very slight displacement of the classical distribution to larger $q$ 's (see Figure 3). This displacement becomes negligible when going from A1 to A3 models (not shown) and thus it should not be related to the real component of the anion PEC, which are the same is these cases. On the other hand, larger shifts on the distribution appear when moving to B1 and C1 models (not shown), in line with the worsening in the corresponding DEA cross sections. This indicates that the shift on the ensemble distribution and consequently on the DEA cross section arises from the different description of the imaginary component. Larger $q$ 's are associated to lower resonance energies, which explains the observed redshift. Still, the comparison between the DEA cross sections (top left panel of Figure 5) is less favorable than the underlying distributions (Figure 2), which suggests that it is not only the imaginary 
component to be blamed for the larger discrepancy of the former. We have found the phase of the quantum wavepacket changes considerably before the stabilization of the anion state, which is obviously absent in the classical propagation. Interference effects should become more pronounced when going from faster (model A1) to slower (model C1) dynamics and could thus play a role in explaining the differences between quantum and mixed quantumclassical DEA cross sections. We have also compared the average resonance energy and width, as computed in both descriptions, and they follow the same trends discussed above.
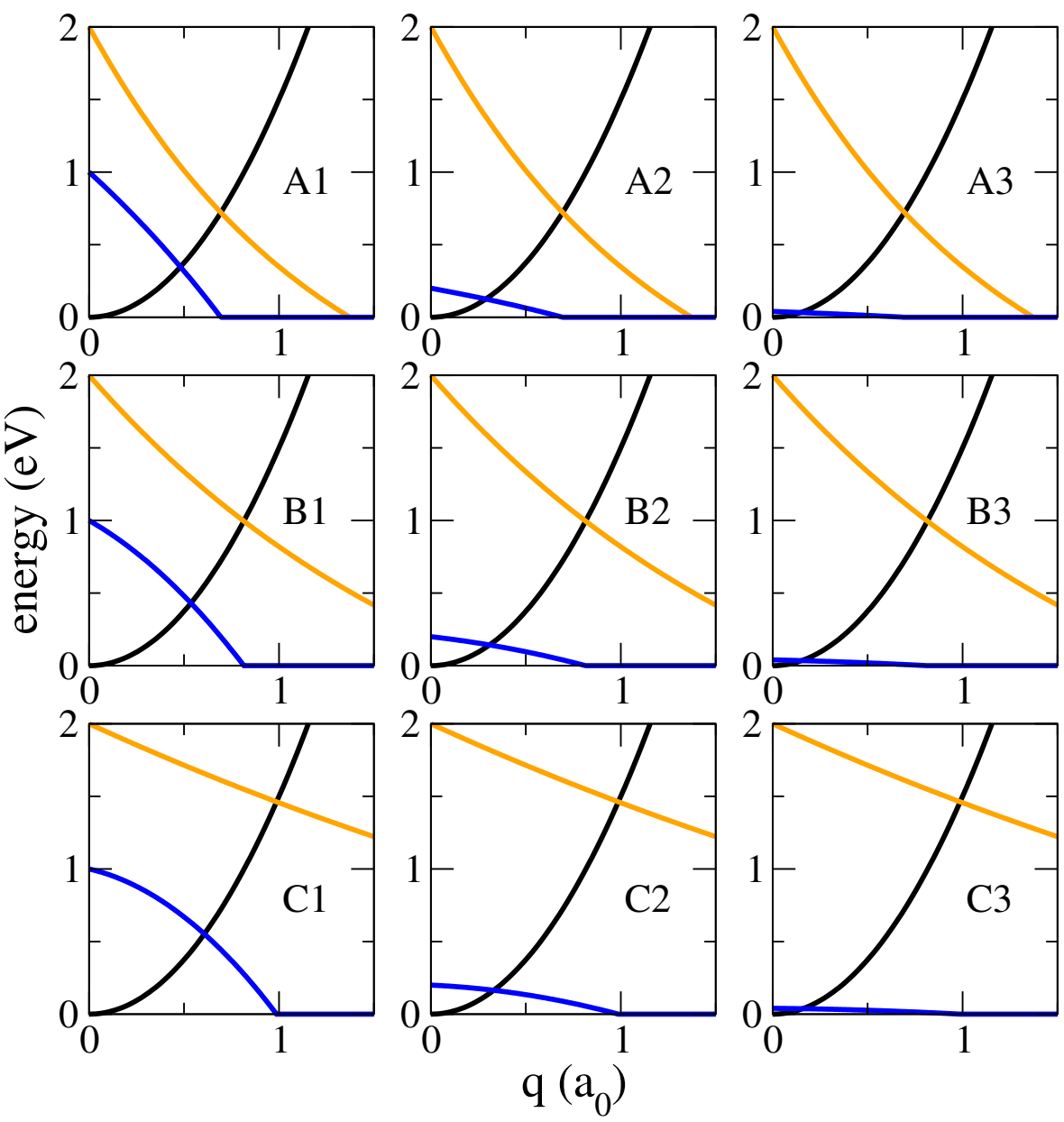

Figure 4: Model PECs (according to eq 24) for the neutral state (black) and for the real (orange) and imaginary (blue) components of the anion state, for $C=1.5 \mathrm{eV}, E_{r}^{0}=2 \mathrm{eV}$ and $D=-1 \mathrm{eV}$. We considered combinations of $\alpha=0.8 a_{0}^{-1}$ (top), $0.5 a_{0}^{-1}$ (middle) and $0.2 a_{0}^{-1}$ (bottom), and $\Gamma^{0}=1.0 \mathrm{eV}$ (left), $0.2 \mathrm{eV}$ (middle) and $0.04 \mathrm{eV}$ (right).

In the following, we briefly compare the results of the nine models. In models A3, B3 and C3, the vertical width assumes a very small value $(0.04 \mathrm{eV})$, such that the survival probabil- 

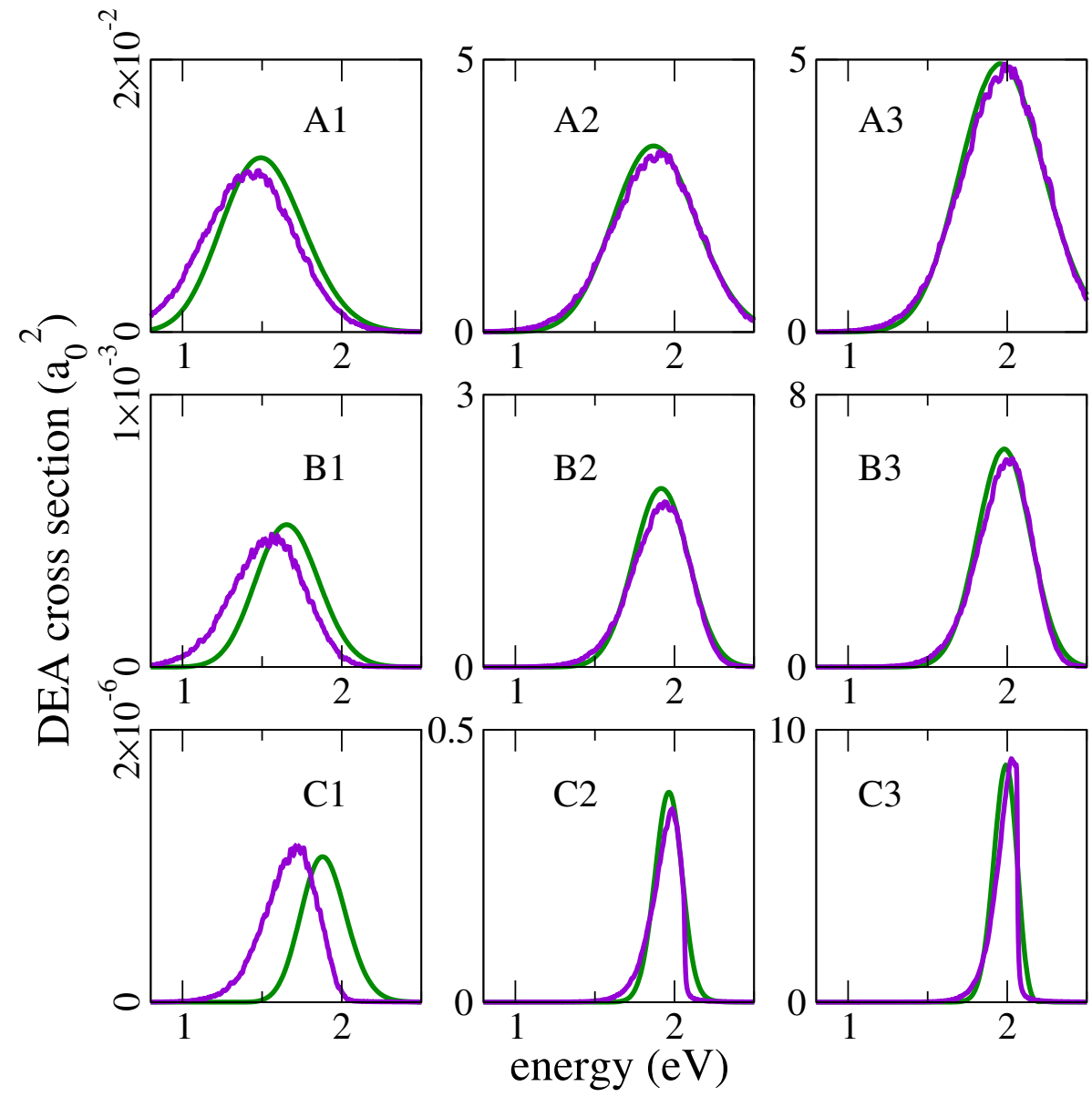

Figure 5: DEA cross section for the model PECs of Figure 4, computed according to quantum (green) and mixed quantum-classic (purple) propagation.

ities are closer to 1 , the DEA cross sections are large and the peak positions coincide with the vertical resonance energy $(2 \mathrm{eV})$. The narrowing of the peak (and larger maximum) when moving from model A3 to C3 reflects the less steep dissociating PEC. The real component $\mathrm{PEC}$ of the anion is the same in models $\mathrm{A} 1, \mathrm{~A} 2$ and $\mathrm{A} 3$, while the vertical resonance widths assume the values 1, 0.2 and $0.04 \mathrm{eV}$. For larger resonance widths, the variation of the survival probabilities on the initial position (and momentum) becomes more dramatic, which means the important region in initial conditions phase space for DEA becomes even more displaced from the equilibrium region. This implies in a shift from the vertical resonance energy to the actual DEA peak position and a decrease in the cross section magnitudes. Indeed, both effects are systematically observed in our model PECs. For a fixed row of Figure 5, the 
comparison along columns shows exactly that. Additionally, we found a larger effect on the cross sections for the slower dynamics (last row), when magnitudes are reduced by six orders of magnitude, compared to the two order of magnitude difference for the faster dissociation (first row). On the other hand, the peak position becomes less displaced when going from faster (first row) to slower (last row) dynamics, which is more marked when comparing A1, $\mathrm{B} 1$ and $\mathrm{C} 1$ models.

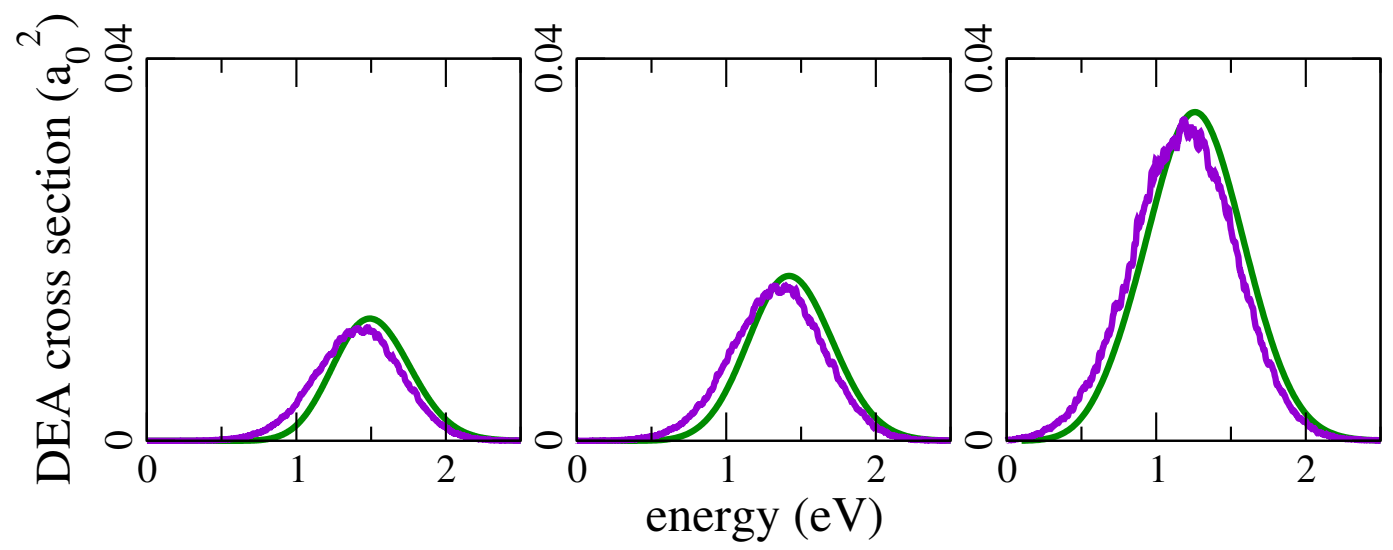

Figure 6: DEA cross section for the model A1, for the neutral state prepared at $0 \mathrm{~K}$ (left), $300 \mathrm{~K}$ (middle) and $500 \mathrm{~K}$ (right).

We have further considered how results for model A1 are affected when preparing the initial conditions at different temperatures: $0 \mathrm{~K}, 300 \mathrm{~K}$ and $500 \mathrm{~K}$. For the quantum propagation, the DEA cross sections were obtained by Boltzmann averaging the contribution from each vibrational level, while in the mixed quantum-classical approach the appropriate finite temperature Wigner distribution was employed. As can be seen in Figure 6, an increase in temperature enhances the cross section and shifts its peak position to smaller energies, which is due to DEA to the vibrationally excited levels of the neutral. The important aspect for our main discussion is that both approaches provide very similar results.

Finally, we have considered different reduced masses: 90 a.m.u., 9 a.m.u. and 1 a.m.u., again for the reference model A1. We found it to have a huge impact on the magnitudes of the cross sections (shown in Figure 7), which spanned 11 orders of magnitude. For a larger reduced mass, the dissociation dynamics takes longer, thus enhancing the probability 
for autodetachment and decreasing the DEA cross section. Moreover, the heavier particle is more localized, which accounts for the narrower peak. Once again, the matching between both descriptions for the nuclear propagation is great, specially when realizing the range of orders of magnitude involved.
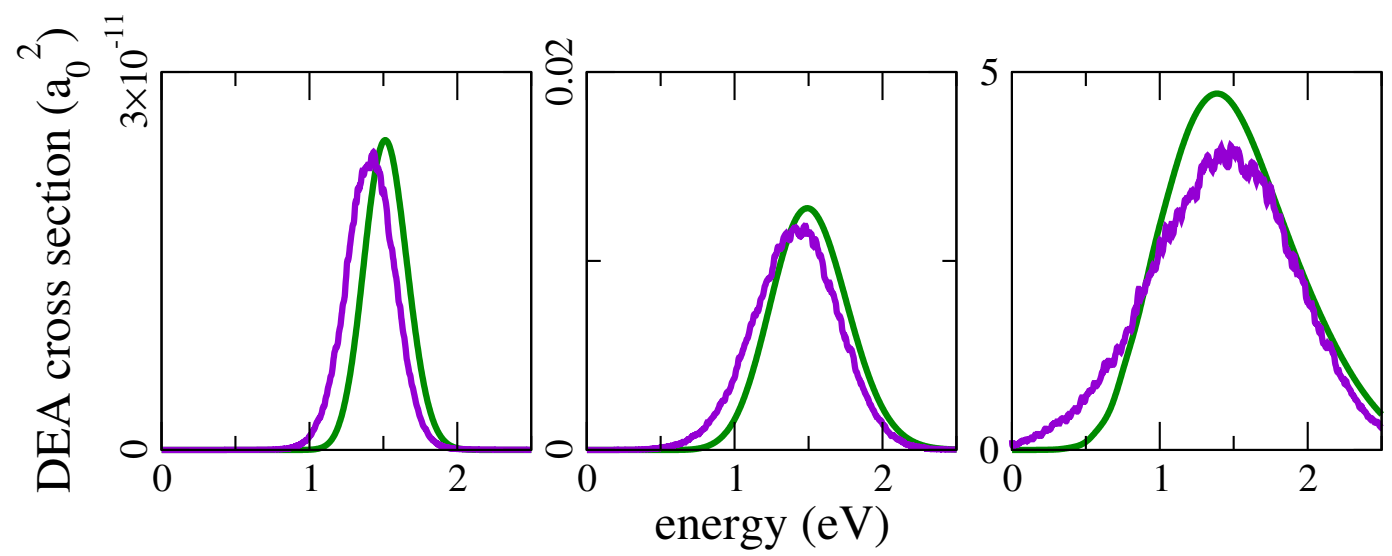

Figure 7: DEA cross section for the model A1, for a reduced mass of 90 a.m.u (left), 9 a.m.u. (middle) and 1 a.m.u. (right).

\section{Chloroethane}

\subsection{Static results}

Elastic integral cross sections of the $\mathrm{A}^{\prime}$ symmetry are shown in Figure 8, together with the computed eigenphase sum, obtained at the neutral equilibrium geometry. The least-squares fit to the later is also shown, according to local (eq 18) and non-local (eq 19) functional forms. We found the vertical resonance energy at $2.33 \mathrm{eV}$, which matches very well with the value obtained from ETS measurements $(2.35 \mathrm{eV}) .{ }^{28}$ This value lies slightly below the elastic cross section peak $(2.43 \mathrm{eV})$, in view of the its considerable width and the influence of background scattering. ${ }^{85}$ Our peak position is also close to what was reported in a previous SMC calculation ${ }^{29}(2.6 \mathrm{eV})$. The vertical resonance width was obtained at $1.35 \mathrm{eV}$, which corresponds to an autodetachment lifetime of 0.49 fs. Our computed width is smaller than the $1.8 \mathrm{eV}$ obtained from the ETS spectrum, ${ }^{31}$ but it should be kept in mind that the 
measured value includes the vibrational broadening of the nuclear wavepacket, absent in our single fixed-nuclei calculation.
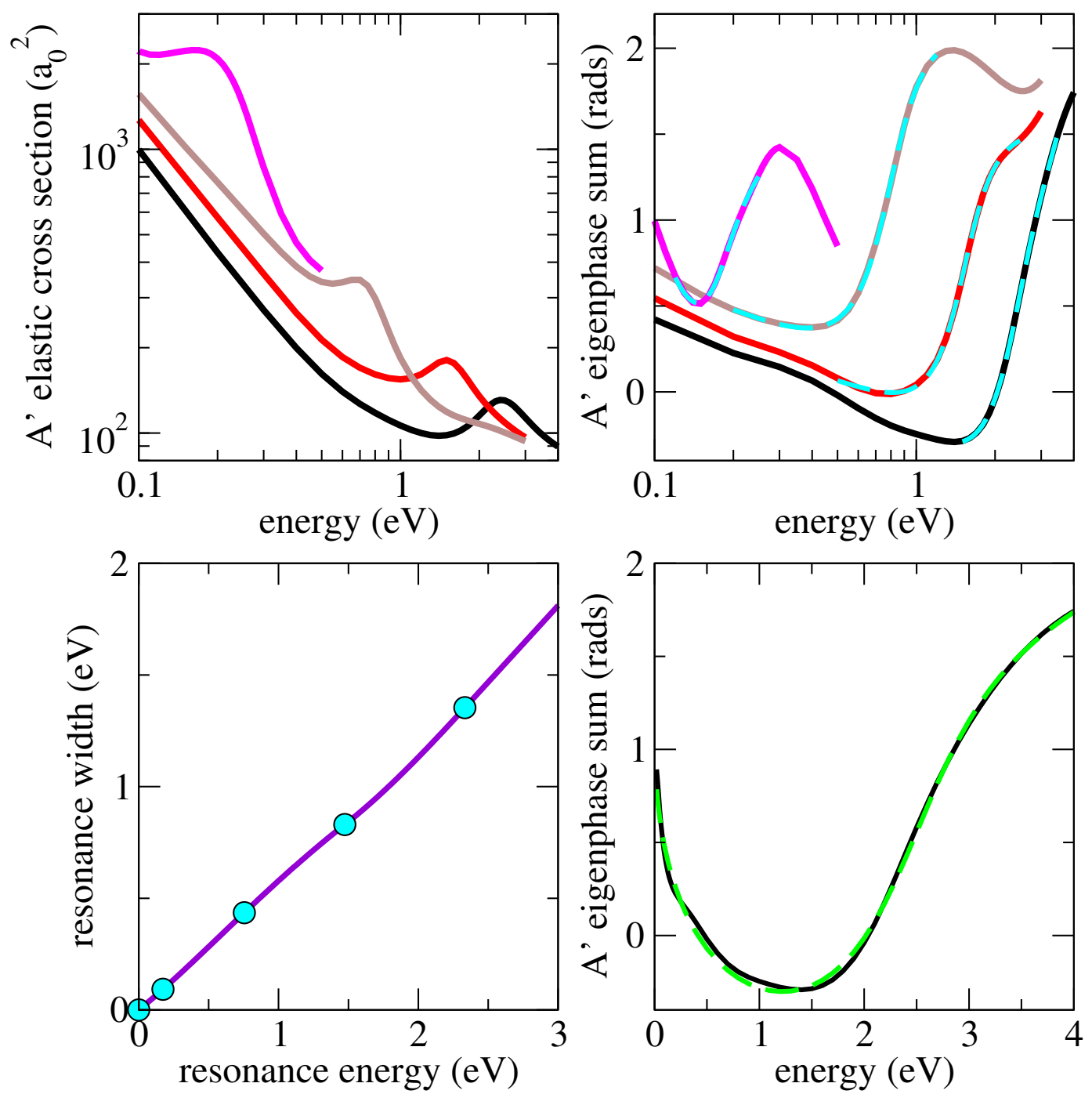

Figure 8: $\mathrm{A}^{\prime}$ elastic cross section (top left) and corresponding eigenphase sum (top right) of chloroethane, with the fitted Breit-Wigner profile (eq 18) in cyan, as computed for the equilibrium geometry (black), and for $\mathrm{C}-\mathrm{Cl}$ stretchings of $0.1 a_{0}$ (red), $0.2 a_{0}$ (brown) and $0.3 a_{0}$ (magenta). In the bottom left, computed pairs of resonance widths and energies (dots) and the interpolated function (violet) employed for the dynamics. In the bottom right, non-local fit (eq 19) (green) to the computed equilibrium geometry eigenphase sum (black).

The distribution of resonance energies at the Franck-Condon region was obtained by performing an additional set of 1,000 MRCISD single point calculations, as sampled from the unshifted harmonic potential Wigner distribution. We had to disregard around $6 \%$ of these points, which grouped at considerably lower resonance energies than the vertical value, thus 
indicating these calculations were actually emulating the continuum. By including them and assigning an arbitrary and common resonance energy of $2.8 \mathrm{eV}$, average and standard deviation of the energy distribution increase very slightly $(0.03 \mathrm{eV})$, and we thus prefer to simply discard these points. Interestingly, the ensemble average of the resonance energy $(2.15 \mathrm{eV})$ lies below the vertical value $(2.33 \mathrm{eV})$. This vibrationally averaged energy should more closely correspond to what is obtained from ETS measurements $(2.37 \mathrm{eV}) .{ }^{28}$ Assuming this $0.18 \mathrm{eV}$ redshift applies for the measured ETS spectrum, the equivalent of an experimental vertical resonance energy would be at $2.55 \mathrm{eV}$. Making use of the importance sampling technique ${ }^{65}$ we found a very close shift $(0.15 \mathrm{eV})$ when the neutral state is prepared at $0 \mathrm{~K}$, while the temperature increase to $333 \mathrm{~K}$ accounts for the remaining $0.03 \mathrm{eV}$. The present finding is somewhat analogous to what is encountered in molecular photoexcitation, where the absorption band maximum is usually redshifted relative to the vertical excitation energy, ${ }^{65,86}$ due to both zero-point and temperature effects. ${ }^{65}$ We are not aware of previous discussions of this effect in the context of transient anions. The distribution of resonance energies has a standard deviation of $0.22 \mathrm{eV}$, which should represent the vibrational broadening width. Adding this number to the computed vertical resonance width of $1.35 \mathrm{eV}$, we obtain a composite energy width for electron attachment of $1.57 \mathrm{eV}$, closer to the measured $1.8 \mathrm{eV}{ }^{28}$

In order to build the autodetachment model employed in the dynamics simulations (eq 11), we have performed additional scattering calculations for geometries where the C$\mathrm{Cl}$ bond was stretched by $0.1,0.2$ and $0.3 a_{0}$, while the other coordinates were kept fixed. The computed elastic cross sections and eigenphase sums (and the fits to the later) are also displayed in Figure 8. We found that the resonance width strongly correlates with the resonance energy. This result is in line with the linear relation between widths and energies found for a series of chloroalkanes in ETS measurements. ${ }^{31,87}$ The interpolation to the pairs of computed resonance width and energy (also displayed in Figure 8) provided the $\Gamma_{L}\left(E_{r}\right)$ function that was employed in the dynamics.

Moving to the results obtained from bound state methods, we found that the vertical 

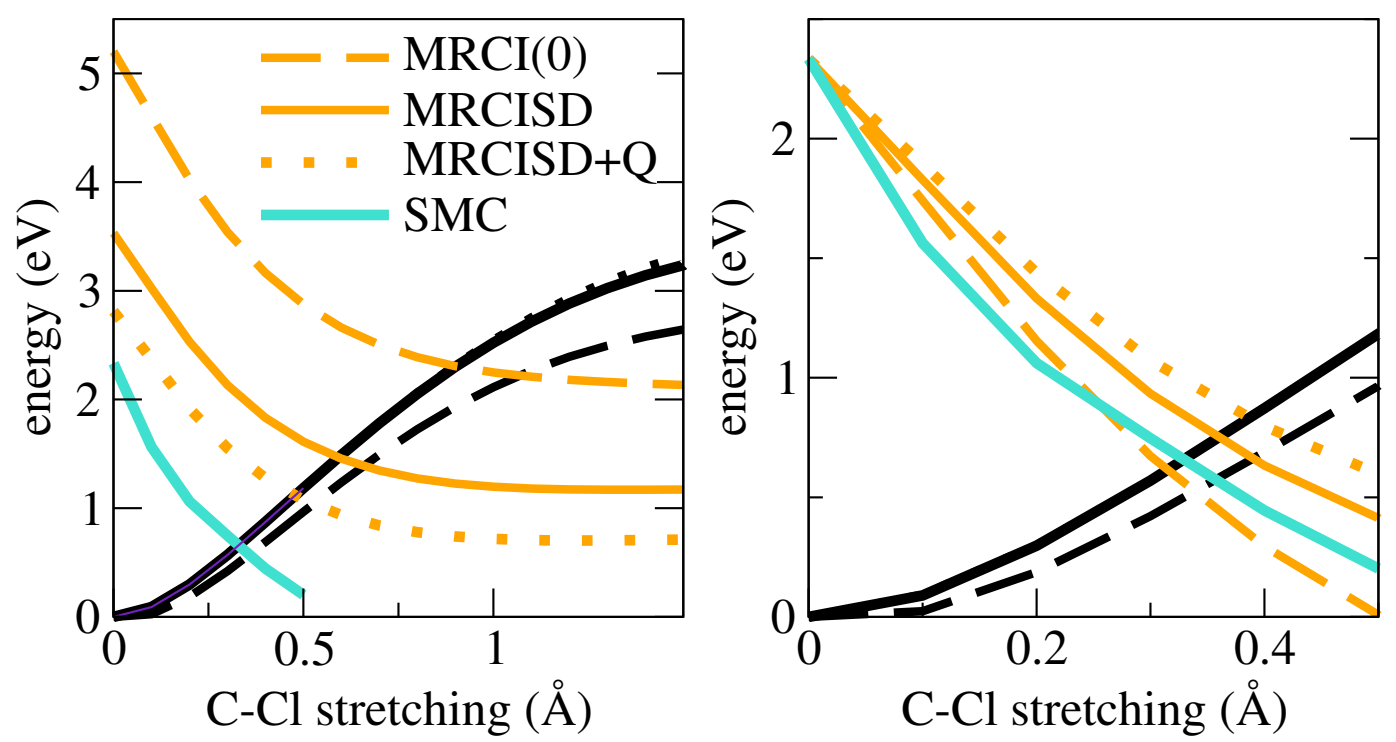

Figure 9: Left: PECs for the neutral ground state (black) and the $\sigma^{*}$ anion state of chloroethane, as computed with different levels of bound state calculations (orange) and with SMC scattering calculations (turquoise). Right: same PECs, but vertically shifted in order to match the SMC vertical energy.

resonance shifts to smaller energies and approaches the experimental value when the level of theory is improved: $4.97 \mathrm{eV}$ with $\mathrm{MRCI}(0), 3.53 \mathrm{eV}$ with MRCISD and $2.81 \mathrm{eV}$ with MRCIS $+\mathrm{Q}$, compared to the measured $2.33 \mathrm{eV} .{ }^{28}$ We assign this discrepancy to an unbalance of correlation effects in the description of anion and neutral states, which is a well known issue. Simply due to the larger number of electrons, there should be more correlation energy in the anion than in the neutral, such that very sophisticated calculations are needed for obtaining quantitative resonance energies. ${ }^{88,89}$ Additionally, very large basis sets are usually needed for calculations of accurate energies. ${ }^{72,88,89}$ but if we were to employ these, a continuum-like state would be described, instead of the valence transient anion. Despite lacking an accurate description of correlation effects, the current implementation of the SMC method provides a more or less balanced description of neutral and anion states, which explains the quite reasonable results for low-lying shape resonances it usually delivers. Figure 9 displays the PECs of the $\sigma^{*}$ resonance and the neutral state of chloroethane, along the $\mathrm{C}-\mathrm{Cl}$ stretching coordinate, as computed in the $\mathrm{MRCI}(0), \mathrm{MRCISD}$ and $\mathrm{MRCISD}+\mathrm{Q}$ levels. It also shows the anion PECs shifted by a fixed amount, such that the vertical resonance at each level of 
calculation matched the SMC result $(2.37 \mathrm{eV})$. The MRCISD PEC, for example, was shifted by $\Delta E_{r, \mathrm{MRCISD}}^{0}=E_{r, \mathrm{SMC}}^{0}-E_{r, \mathrm{MRCISD}}^{0}$, where $E_{r, \mathrm{SMC}}^{0}=2.37 \mathrm{eV}$ and $E_{r, \mathrm{MRCISD}}^{0}=3.53 \mathrm{eV}$ are the vertical resonance energies obtained with the SMC and MRCISD calculations. This correction compensates for the lack of correlation effects and the limited basis set employed in the bound state calculations for the anion. Since the PECs computed with both bound state and scattering calculations are roughly parallel, it is reasonable to assume the same correction could be applied beyond the neutral equilibrium geometry. Therefore, this correction was imposed on top of the anion $\left(V_{d}(R)\right)$ and neutral $\left(V_{0}(R)\right)$ energies computed along the dynamics simulation, such that the on-the-fly resonance energies were actually evaluated as $E_{r}(R)=V_{d}(R)-V_{0}(R)+\Delta E_{r, \text { MRCISD }}^{0}$. Similarly, the resonance widths were computed at each timestep as described in the bottom left panel of Fig. 8.

\subsection{Dynamics results}

The evolution of the resonance energy for 200 out of the 1,400 trajectories (for clarity reasons) is shown in Figure 10, together with the whole ensemble average for both resonance energy and width. It also shows the survival probability, importance sampling weight and the DEA cross section contribution of each trajectory, plotted as a function of impact energy. We found that the electronic stabilization of the anion takes place extremely fast, as the average resonance energy goes to zero in 8 fs after electron attachment. Most of the trajectories start with a resonance energy below the vertical value, which is a consequence of the shifted PDF from which the initial conditions were sampled. Trajectories that begun at higher energies have overall large resonance widths and thus their survival probabilities decrease very rapidly, while those starting at lower energies have smaller resonance widths, hence survival probabilities that decrease at a slower rate. Therefore, the later will gain in relative importance compared to the former as the dynamics unfolds. We found a huge variation of the survival probabilities, which span 9 orders of magnitude and decrease very rapidly as the initial resonance energy increases. On the other hand, the different sampling and 

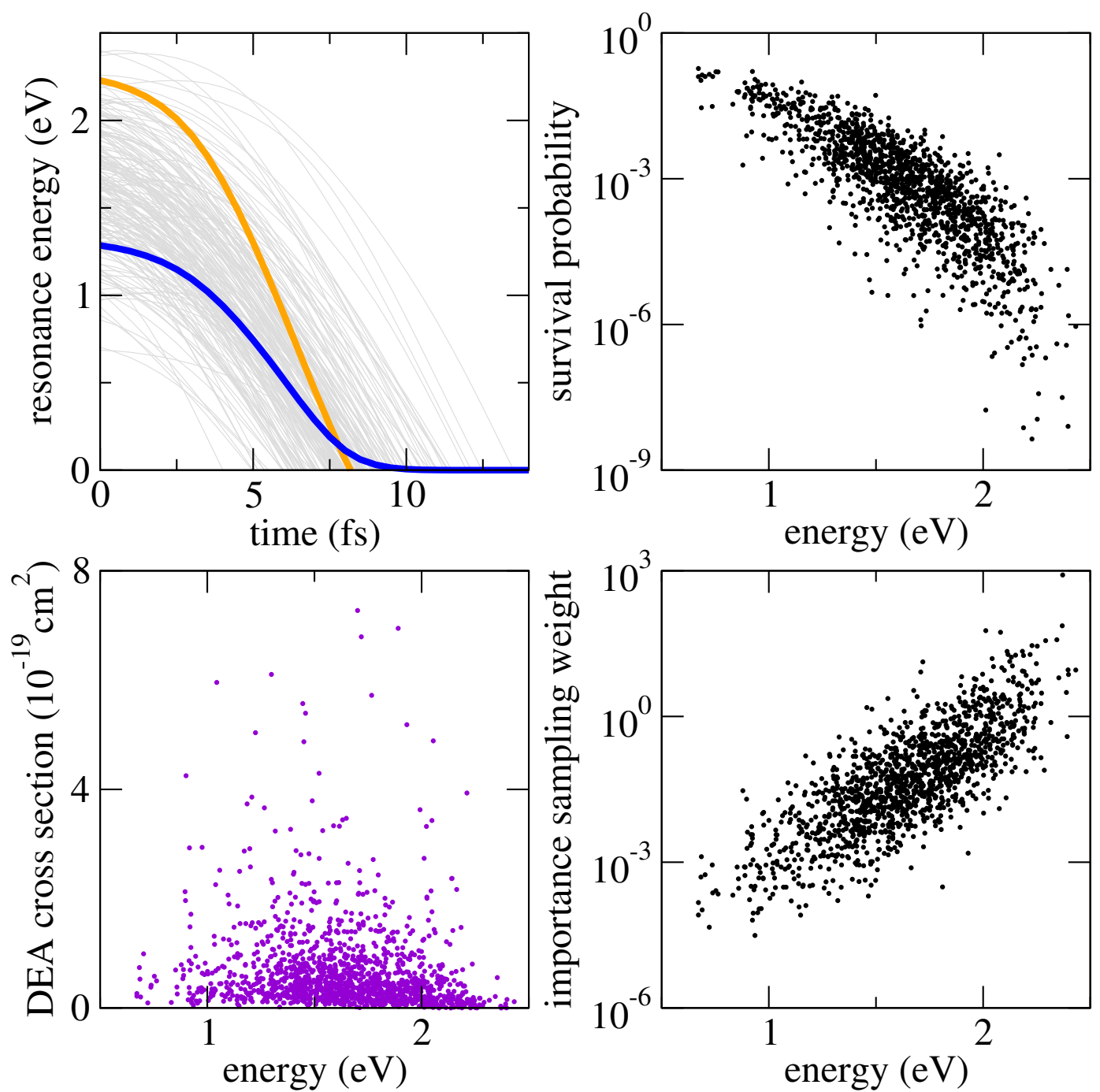

Figure 10: Top left: resonance energy for 200 out of the 1,400 trajectories (gray), and ensemble average resonance energy (orange) and width (blue). On the other panels, survival probability, importance sampling weight and DEA cross section for each of the 1,400 trajectories.

target PDFs lead to importance sampling weights that correlate positively with the initial resonance energy. Both survival probabilities and importance sampling weights are accounted for when computing the contribution of each trajectory to the DEA cross section, such that the effective weight becomes much more uniform, as shown in the bottom left panel of Figure 10. The uniformity of these points means that there is a good overlap between the sampling PDF and the region in phase space which is most important for the DEA process.

Figure 11 shows the evolution of the four most activated internal coordinates, for the 

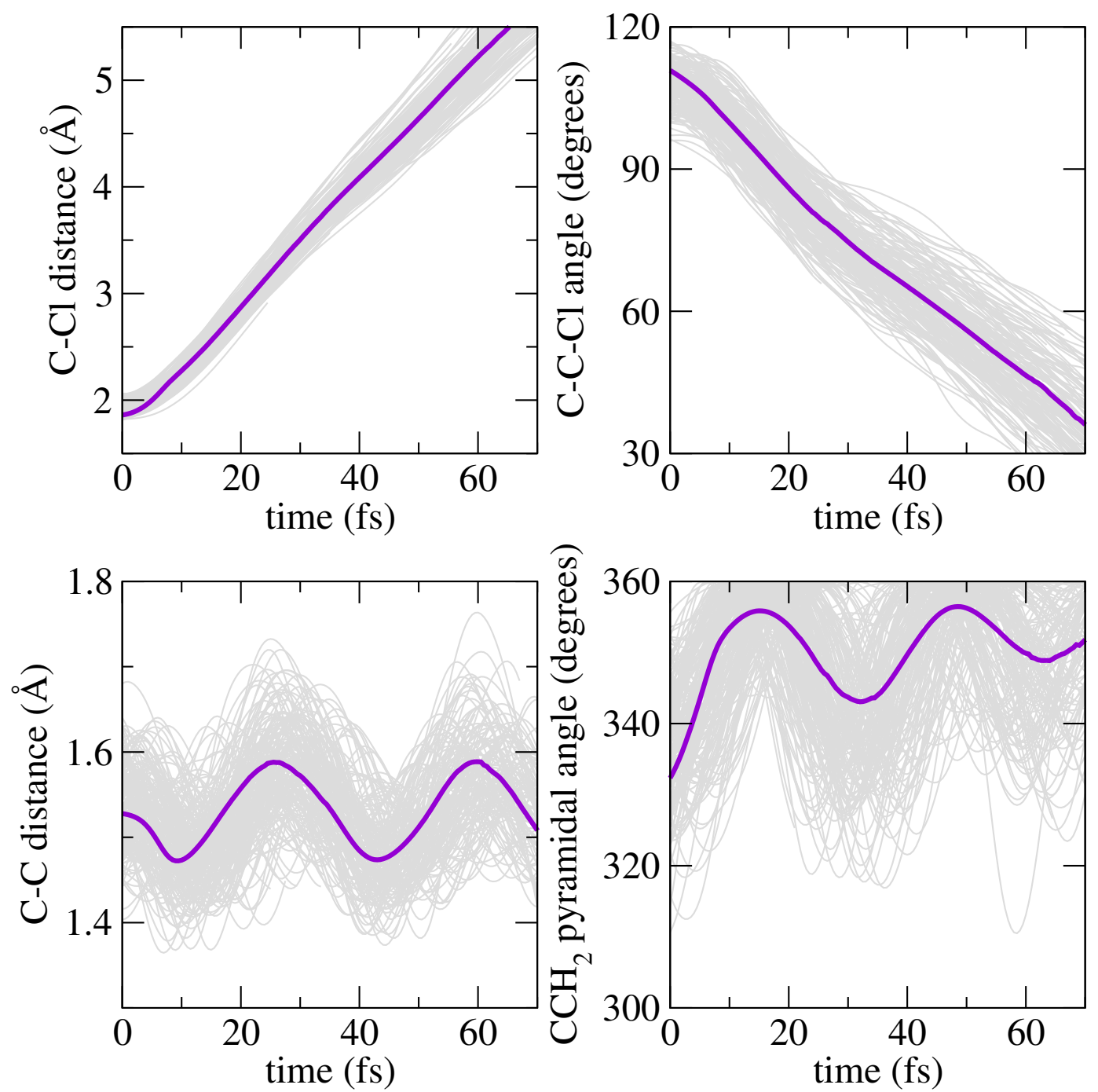

Figure 11: Evolution of four internal coordinates for each trajectory (gray) and for the ensemble average (violet). The coordinates are the $\mathrm{C}-\mathrm{Cl}$ distance, the $\mathrm{C}-\mathrm{C}$ distance, the $\mathrm{C}-\mathrm{C}-\mathrm{Cl}$ angle and the $\mathrm{CCH}_{2}$ pyrimidalization angle, defined as the average between the three bending angles to the central carbon atom.

ensemble average and for the 200 trajectories that run beyond the $4.5 a_{0} \mathrm{C}-\mathrm{Cl}$ distance threshold. The dynamics simulation reveals that the electron capture into the $\sigma^{*}$ orbital readily induces the stretching of the $\mathrm{C}-\mathrm{Cl}$ bond, as the departing chloride ion gains momentum to dissociate. As this is the dominant vibrational relaxation mechanism, most of the impact energy should be primarily transfered to kinetic energy of the fragments when DEA takes place. On top of that, this result supports the autodetachment model we have adopted, which only considered the behavior of the resonance width upon the $\mathrm{C}-\mathrm{Cl}$ stretching. Ad- 
ditional scattering calculations (not shown) further indicate that the width is less sensitive to the activation of other vibrational modes. Figure 11 further shows the reaction of other internal coordinates to the electron capture and to the $\mathrm{C}-\mathrm{Cl}$ stretching. The recoil of the departing chloride ion induces a shortening of the $\mathrm{C}-\mathrm{C}$ bond, which continues to oscillate coherently after dissociation (at least in the first $100 \mathrm{fs}$ ). The $\mathrm{C}-\mathrm{C}-\mathrm{Cl}$ angle is shortened in a more or less constant rate, which indicates that the neutral fragment gains some rotation. Also, the $\mathrm{CH}_{2}$ hydrogens initially move towards the halogen atom, which causes the $\mathrm{CCH}_{2}$ moiety to oscillate around its planar conformation. Thus, our results point out that the $\mathrm{CH}_{3} \mathrm{CH}_{2}$ fragment should be formed with some rotational excitation and in vibrationally excited levels for the $\mathrm{C}-\mathrm{C}$ stretching and the $\mathrm{CH}_{2}$ wagging modes.

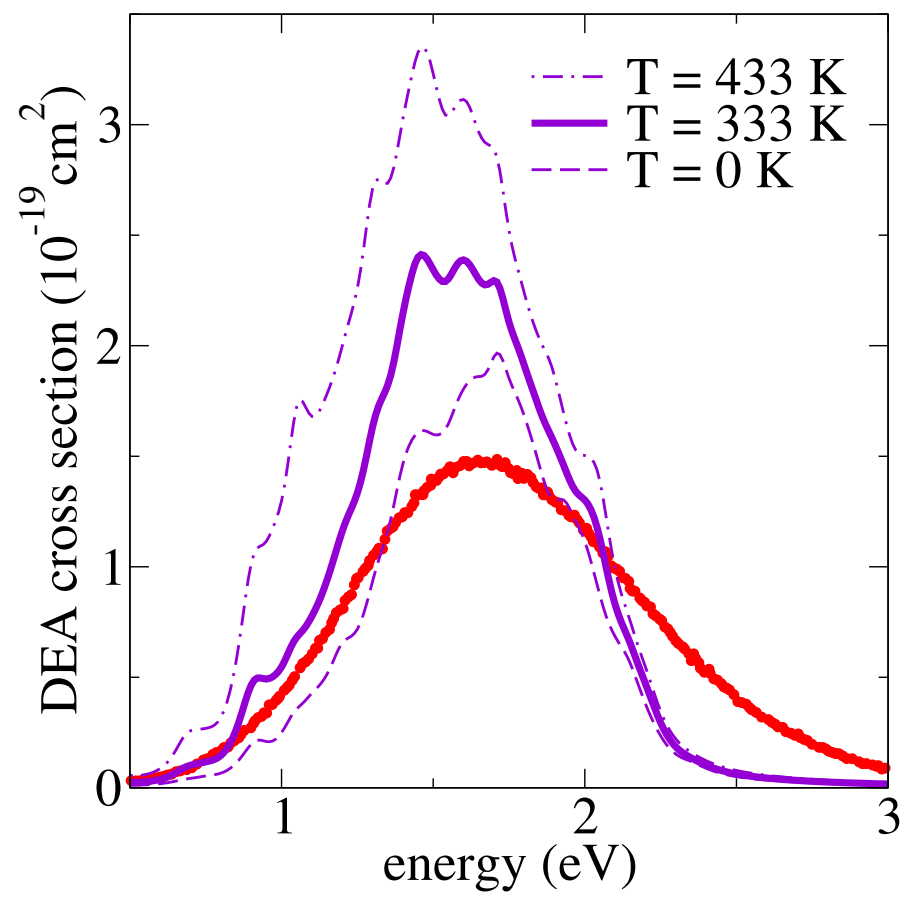

Figure 12: Computed DEA cross section of chloroethane (violet), for the neutral state prepared at $0 \mathrm{~K}, 333 \mathrm{~K}$ (experimental condition) and $433 \mathrm{~K}$. Measured data ${ }^{31}$ in red.

Our computed DEA cross section is presented in Figure 12, together with the measured data of Pearl and Burrow. ${ }^{31}$ In their first measurement, ${ }^{30}$ the ion yield peaked at $1.7 \mathrm{eV}$, with a cross section of $(1.6 \pm 0.3) \times 10^{-19} \mathrm{~cm}^{2}$ while in a subsequent paper, ${ }^{31}$ they report a maximum DEA cross section of $1.49 \times 10^{-19} \mathrm{~cm}^{2}$, peaking at $1.49 \mathrm{eV}$. The later data 
was reproduced in Figure 2 of Ref., ${ }^{87}$ from which we extracted the data points presented in Figure 12. Our calculations correctly reproduce the shift from the vertical resonance energy $(2.37 \mathrm{eV})$ to the peak position of the DEA cross section $(1.56 \mathrm{eV})$, which agrees very well to the measured displacement from $2.33 \mathrm{eV}$ to $1.49 \mathrm{eV}$. Furthermore, the DEA cross section magnitudes are remarkably close to experiment.

The recently proposed application of the importance sampling technique for dynamics simulations allows for an inexpensive evaluation of temperature effects on computed observables. ${ }^{65}$ We have thus assessed temperature effects on the DEA cross section of chloroethane, which is also presented in Figure 12. Heating of the neutral molecule is followed by a sizable increase in the cross section and a small shift of the peak position to lower energies. This finding is consistent with previous reports on the effect of temperature on DEA induced by short-lived resonant states. ${ }^{90-92}$ Even for the rather small variation from room temperature $(288 \mathrm{~K})$ to the temperature of the experiment $(333 \mathrm{~K})$, we found a non-negligible $10 \%$ increase in the DEA cross section magnitude (not shown).

\subsection{Precision and accuracy assessment}

In the following, we discuss how the computed DEA cross section is modified when precision and accuracy of the underlying calculations are varied. Figure 13 shows that with as few as 100 trajectories, the curves are already quite close to that obtained with the much larger set of 1,400 trajectories. When the broadening parameter $\eta$ is increased from $0.1 \mathrm{eV}$ to 0.5 $\mathrm{eV}$, the structures in the curves (which arise from the limited number of trajectories) are smoothed out. In the present case, using a larger broadening parameter is justifiable, since the measured cross section is already relatively broad in energy. This is very promising for future applications of the method, where semi-quantitative magnitudes and peak positions are expected to be attained with an affordable number of trajectories. We further evaluated the effect of scaling the MRCISD computed vibrational frequencies by a factor of 0.95, again with the aid of importance sampling calculations. ${ }^{65}$ The DEA cross section (not shown) 


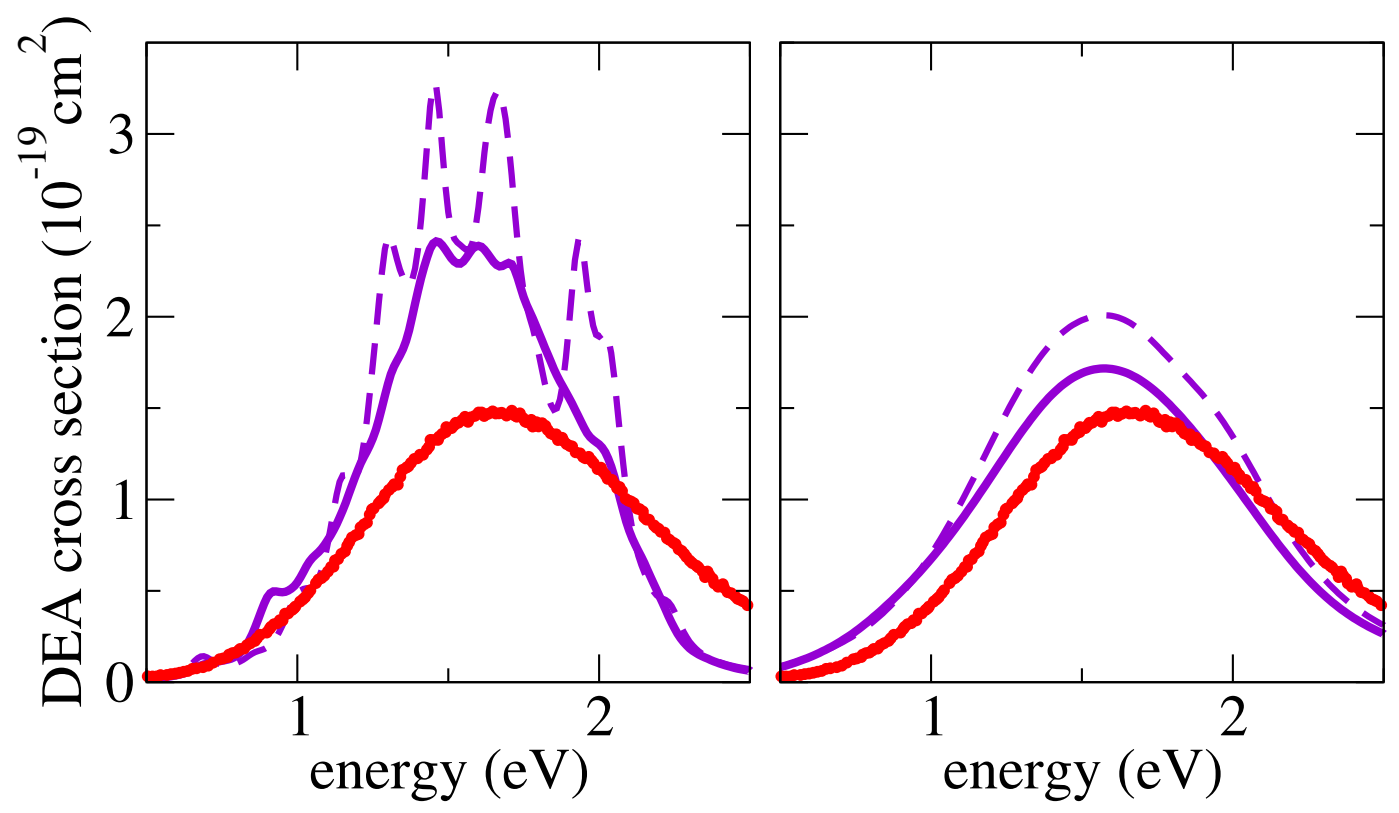

Figure 13: Computed DEA cross section for 1,400 (full lines) and 100 (dashed lines) trajectories, for linewidths of $\eta=0.1 \mathrm{eV}$ (left) and $\eta=0.5 \mathrm{eV}$ (right). Measured data ${ }^{31}$ in red.

increased by only $5 \%$, while the peak remained at the same position.

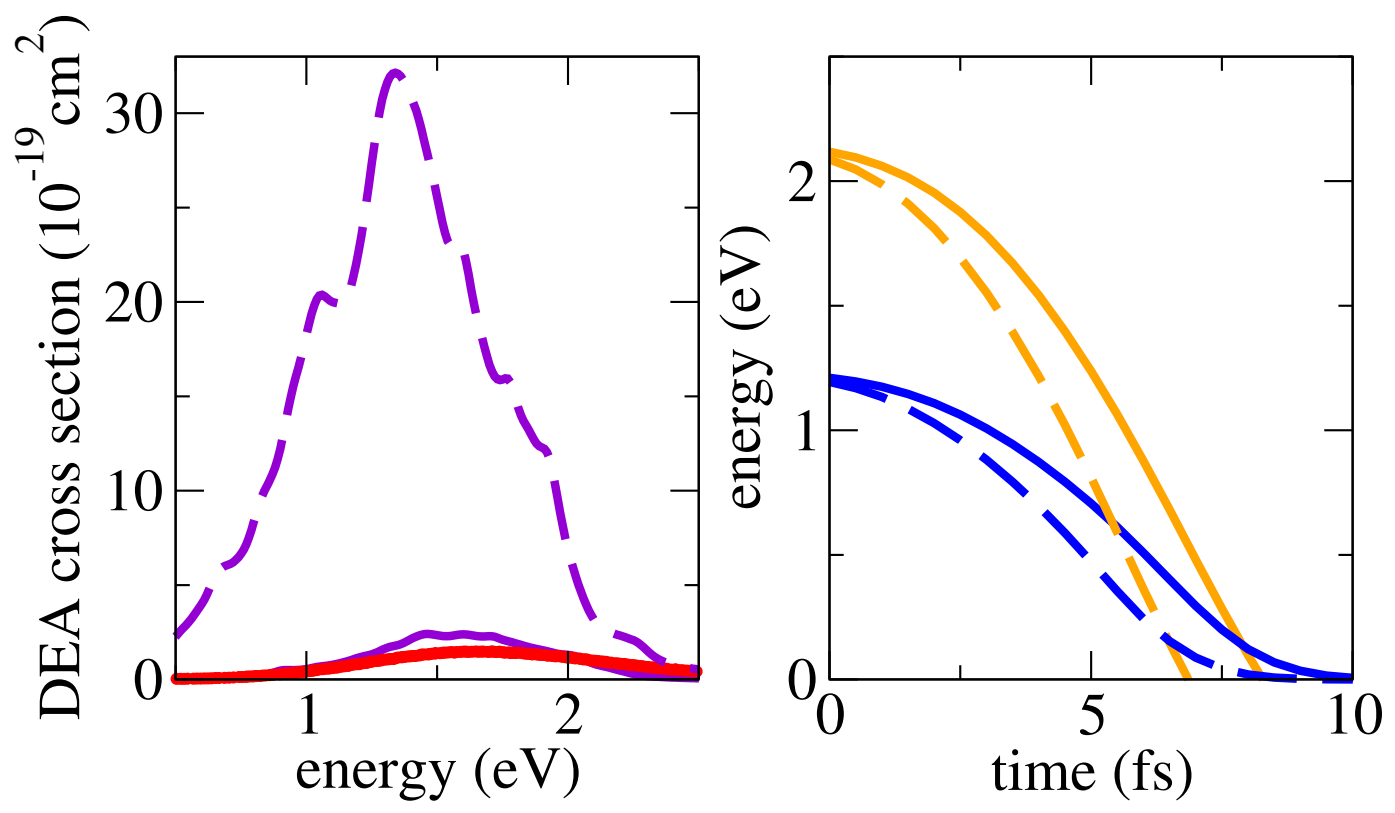

Figure 14: On the left, computed DEA cross section of chloroethane, according to MRCISD (full violet) and $\mathrm{MRCI}(0)$ (dashed violet) dynamics, together with the measured data ${ }^{31}$ (red). On the right, ensemble average of the resonance energy (orange) and width (blue), for the two levels of theory (full and dashed lines). 
In Figure 14 we compare DEA cross sections computed with both MRCISD and MRCI(0) levels of description for the transient anion dynamics. MRCI(0) results provide one order of magnitude larger cross sections, peaking at even lower energies $(1.34 \mathrm{eV})$, when compared to the more accurate MRCISD results. This is due to the faster stabilization of the resonance energy (and width), also compared in Figure 14, which in turn reflect the steeper MRCI(0) PEC (see Figure 9).

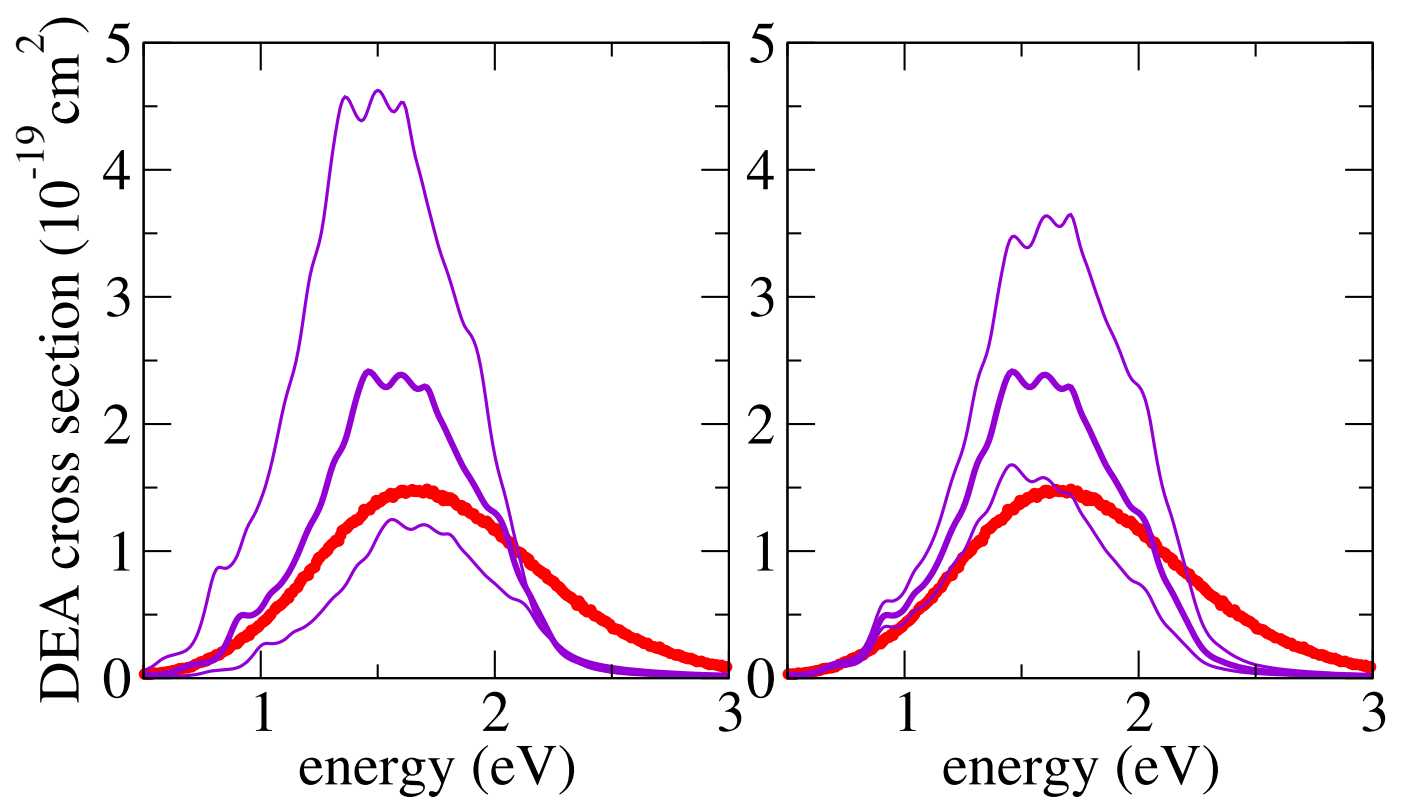

Figure 15: Computed DEA cross section of chloroethane. On the left, curves from top to bottom correspond to increasing vertical resonance energies: 2.23, 2.33 and $2.43 \mathrm{eV}$, while keeping a proportional resonance width. On the right, curves from top to bottom correspond to increasing vertical resonance widths: $1.25,1.35$ and $1.45 \mathrm{eV}$, while keeping the same vertical resonance energy of $2.33 \mathrm{eV}$. Measured data ${ }^{31}$ in red.

Finally, we also assessed the sensitivity of DEA cross sections on the vertical resonance energy and width. Two cases were tested: (i) a $\pm 0.1 \mathrm{eV}$ variation of the vertical resonance energy, while the vertical widths also change accordingly, and (ii) same vertical resonance energy, but vertical widths modified by $\pm 0.1 \mathrm{eV}$. Results of this comparison, shown in Figure 15, point out that the DEA cross sections are rather sensitive to both vertical energy and width. The magnitudes are impacted by $\sim 50 \%$ when the width varies by only $0.1 \mathrm{eV}$, while the same shift in the resonance energy brings in a factor of 2 to the cross section. These 
scenarios should be representative of what one encounters in scattering calculations. For example, typical precisions of resonance energies obtained with the SMC method are around $\sim 0.3 \mathrm{eV}$. When a resonance is found in the upper limit of this margin, its width tends to follow accordingly and be wider than if it had been found in the lower limit, which contemplates case (i). Besides that, scattering calculations may provide comparable resonance energies and yet rather distinct widths, as recently discussed for the low-lying resonances of para-benzoquinone. ${ }^{93}$ We have performed an additional set of scattering calculations for chloroethane, without the additional set of very diffuse $4 \mathrm{~s} 3 \mathrm{p}$ functions (not shown). Even though the resonance positions were not affected considerably, the linear correlation between resonance widths and energies (upon $\mathrm{C}-\mathrm{Cl}$ stretching) turned out to be noisier than what was found when including the extra $4 \mathrm{~s} 3 \mathrm{p}$ diffuse set. This results suggests that calculating accurate resonance widths might require more diffuse functions that are usually employed for describing resonance energies.

\section{Conclusions}

A novel theoretical framework for describing the dynamics of transient anions has been proposed. It is based on propagating an ensemble of classical trajectories, where resonance energies are computed on-the-fly with bound state methods, while the widths are described with a model that requires few electron scattering calculations. It has been implemented into the Newton-X package, and will be freely available to the community in the next release of the code. The proposed mixed quantum-classical dynamics approach was benchmarked against quantum dynamics propagations, for model one dimensional potential energy curves. Different slopes of the dissociating curve, resonance widths, temperatures and reduced masses were considered, and both methodologies consistently delivered quite close DEA cross section curves.

The very first application of the proposed methodology to a real molecular target con- 
cerned the dissociation of chloroethane induced by electron capture. While autodetachment is the main decaying mechanism, we found it only takes place at very short timescales $(\sim 10$ fs), since the $\mathrm{C}-\mathrm{Cl}$ stretching rapidly stabilizes the $\sigma^{*}$ transient anion. The fragments should be formed with high kinetic energy, while some of the deposited energy should be transfered to vibrational excitation of the neutral radical. The computed DEA cross section curve is in excellent agreement to existing experimental data, both in magnitude and in shape. In particular, our calculations provide a quantitative shift from the vertical resonance energy to the ion yield peak energy. We also found the cross section to be quite temperature dependent.

When accounting for rather conservative uncertainties on the resonance energy and width, DEA cross sections varied to within one order of magnitude. This great sensitivity highlights the need for further theoretical developments on the description of transient anions. Special attention should be given to the widths of resonant anions, as the uncertainties of calculations are not well known. Furthermore, the cross sections showed an even more pronounced sensitivity on the nuclear energy gradients, which ultimately govern the dissociation time. Therefore, for DEA processes involving short-lived resonances, quantitative cross section magnitudes require very sophisticated electronic structure methods for the dynamics propagation and accurate models for the autodetachment probability. In spite of that, the present application of the proposed methodology provided quantitative observables and a detailed picture of the electron-induced dynamics.

\section{Acknowledgement}

FK acknowledges São Paulo Research Foundation (FAPESP), under grants No. 2015/237925 and 2016/21880-7. MTNV also acknowledges FAPESP, grant No. 2017/24145-9. FK and

MB thank the support of the Excellence Initiative of Aix-Marseille University (A*MIDEX) and the project Equip@Meso (ANR-10-EQPX-29-01), both funded by the French Government "Investissements d'Avenir" program. They also acknowledge funding from the WSPLIT 
project (ANR-17-CE05-0005-01).

\section{References}

(1) Chourou, S.; Orel, A. Dissociative electron attachment to HCN and HNC. Phys. Rev. A 2009, 80, 32709 .

(2) Christophorou, L. G.; Olthoff, J. K. Electron interactions with plasma processing gases: present status and future needs. Appl. Surf. Sci. 2002, 192, 309-326.

(3) Taherzadeh, M. J.; Karimi, K. Pretreatment of lignocellulosic wastes to improve ethanol and biogas production: a review. Int. J. Mol. Sci. 2008, 9, 1621-51.

(4) Arumainayagam, C. R.; Lee, H.-L.; Nelson, R. B.; Haines, D. R.; Gunawardane, R. P. Low-energy electron-induced reactions in condensed matter. Surf. Sci. Rep. 2010, 65, $1-44$.

(5) Thorman, R. M.; Kumar T. P., R.; Fairbrother, D. H.; Ingólfsson, O. The role of low-energy electrons in focused electron beam induced deposition: four case studies of representative precursors. Beilstein J. Nanotechnol. 2015, 6, 1904-1926.

(6) Pshenichnyuk, S. A.; Lomakin, G. S.; Modelli, A. Degradation of gas phase decabromodiphenyl ether by resonant interaction with low-energy electrons. Phys. Chem. Chem. Phys. 2011, 13, 9293.

(7) Luo, J.; Hu, J.; Wei, X.; Fu, L.; Li, L. Dehalogenation of persistent halogenated organic compounds: A review of computational studies and quantitative structure-property relationships. Chemosphere 2015, 131, 17-33.

(8) Gorfinkiel, J. D.; Ptasinska, S. Electron scattering from molecules and molecular aggregates of biological relevance. J. Phys. B: At. Mol. Opt. Phys. 2017, 50, 182001. 
(9) Boudaïffa, B.; Cloutier, P.; Hunting, D.; Huels, M. A.; Sanche, L. Resonant formation of DNA strand breaks by low-energy (3 to $20 \mathrm{eV}$ ) electrons. Science 2000, 287, 1658-60.

(10) Abdoul-Carime, H.; Huels, M. A.; Brüning, F.; Illenberger, E.; Sanche, L. Dissociative electron attachment to gas-phase 5-bromouracil. J. Chem. Phys. 2000, 113, 2517.

(11) Tanzer, K.; Feketeová, L.; Puschnigg, B.; Scheier, P.; Illenberger, E.; Denifl, S. Reactions in Nitroimidazole Triggered by Low-Energy (0-2 eV) Electrons: Methylation at N1-H Completely Blocks Reactivity. Angew. Chem. Int. Ed. Engl. 2014, 53, 12240-3.

(12) Pshenichnyuk, S. a.; Elkin, Y. N.; Kulesh, N. I.; Lazneva, E. F.; Komolov, A. S. Lowenergy electron interaction with retusin extracted from Maackia amurensis: towards a molecular mechanism of the biological activity of flavonoids. Phys. Chem. Chem. Phys. 2015, 17, 16805-16812.

(13) Pshenichnyuk, S. A.; Komolov, A. S. Dissociative electron attachment to resveratrol as a likely pathway for generation of the $\mathrm{H}_{2}$ antioxidant species inside mitochondria. $J$. Phys. Chem. Lett. 2015, 6, 1104-1110.

(14) Domcke, W. Theory of resonance and threshold effects in electron-molecule collisions: The projection-operator approach. Phys. Rep. 1991, 208, 97-188.

(15) Houfek, K.; Rescigno, T.; McCurdy, C. Probing the nonlocal approximation to resonant collisions of electrons with diatomic molecules. Phys. Rev. A 2008, 77, 012710.

(16) Gallup, G. A.; Xu, Y.; Fabrikant, I. I. Nonlocal theory of dissociative electron attachment to $\mathrm{H}_{2}$ and HF molecules. Phys. Rev. A 1998, 57, 2596-2607.

(17) Tarana, M.; Houfek, K.; Horáček, J.; Fabrikant, I. I. Dissociative electron attachment and vibrational excitation of $\mathrm{CF}_{3} \mathrm{Cl}$ : Effect of two vibrational modes revisited. Phys. Rev. A 2011, 84, 052717. 
(18) Haxton, D. J.; McCurdy, C. W.; Rescigno, T. N. Dissociative electron attachment to the $\mathrm{H}_{2} \mathrm{O}$ molecule I. Complex-valued potential-energy surfaces for the ${ }^{2} B_{1},{ }^{2} A_{1}$, and ${ }^{2} B_{2}$ metastable states of the water anion. Phys. Rev. A 2007, 75, 012710.

(19) Haxton, D. J.; Rescigno, T. N.; McCurdy, C. W. Dissociative electron attachment to the $\mathrm{H}_{2} \mathrm{O}$ molecule. II. Nuclear dynamics on coupled electronic surfaces within the local complex potential model. Phys. Rev. A 2007, 75, 012711.

(20) Moradmand, A.; Slaughter, D. S.; Haxton, D. J.; Rescigno, T. N.; McCurdy, C. W.; Weber, T.; Matsika, S.; Landers, A. L.; Belkacem, A.; Fogle, M. Dissociative electron attachment to carbon dioxide via the ${ }^{2} \Pi_{u}$ shape resonance. Phys. Rev. A 2013, 88, 032703.

(21) Gallup, G. A.; Fabrikant, I. I. Vibrational Feshbach resonances in dissociative electron attachment to uracil. Phys. Rev. A 2011, 83, 012706.

(22) Wang, X.-D.; Xuan, C.-J.; Feng, W.-L.; Tian, S. X. Dissociative electron attachments to ethanol and acetaldehyde: A combined experimental and simulation study. J. Chem. Phys. 2015, 142, 064316.

(23) Feng, W.-L.; Tian, S. X. Ab Initio Molecular Dynamics Simulation Study of Dissociative Electron Attachment to Dialanine Conformers. J. Phys. Chem. A 2015, 119, 18381845.

(24) Ásgeirsson, V.; Bauer, C. A.; Grimme, S. Unimolecular decomposition pathways of negatively charged nitriles by ab initio molecular dynamics. Phys. Chem. Chem. Phys. 2016, 18, 31017-31026.

(25) Fennimore, M. A.; Matsika, S. Core-excited and shape resonances of uracil. Phys. Chem. Chem. Phys. 2016, 18, 30536-30545. 
(26) Fennimore, M. A.; Karsili, T. N. V.; Matsika, S. Mechanisms of H and CO loss from the uracil nucleobase following low energy electron irradiation. Phys. Chem. Chem. Phys. 2017, 19, 17233-17241.

(27) Olthoff, J. K.; Tossell, J. A.; Moore, J. H. Electron attachment by haloalkenes and halobenzenes. J. Chem. Phys. 1985, 83, 5627.

(28) Guerra, M.; Jones, D.; Distefano, G.; Scagnolari, F.; Modelli, A. Temporary anion states in the chloromethanes and in monochloroalkanes. J. Chem. Phys. 1991, 94, 484.

(29) Sakaamini, A.; Navarro, C.; Cross, J.; Hargreaves, L. R.; Khakoo, M. A.; Fedus, K.; Winstead, C.; McKoy, V. Low-energy elastic electron scattering form chloroethane, $\mathrm{C}_{2} \mathrm{H}_{5}$ Cl. J. Phys. B: At. Mol. Opt. Phys. 2015, 48, 205202.

(30) Pearl, D.; Burrow, P. Thermal decomposition and the apparent dissociative attachment cross section of heated methyl-, ethyl- and t-butyl-chloride. Chem. Phys. Lett. 1993, 206, 483-487.

(31) Pearl, D. M.; Burrow, P. D. Dissociative attachment in selected monochloroalkanes. J. Chem. Phys. 1994, 101, 2940.

(32) Aflatooni, K.; Gallup, G.; Burrow, P. Dissociative electron attachment in chloroalkanes and the correlation with vertical attachment energies. Chem. Phys. Lett. 1998, 282, 398-402.

(33) Aflatooni, K.; Burrow, P. Dissociative electron attachment in chlorofluoromethanes and the correlation with vertical attachment energies. Int. J. Mass Spectrom. 2001, 205, $149-161$.

(34) Aflatooni, K.; Burrow, P. D. Total cross sections for dissociative electron attachment in dichloroalkanes and selected polychloroalkanes: The correlation with vertical attachment energies. J. Chem. Phys. 2000, 113, 1455. 
(35) Paranjothy, M.; Sun, R.; Zhuang, Y.; Hase, W. L. Direct chemical dynamics simulations: coupling of classical and quasiclassical trajectories with electronic structure theory. WIREs: Comput. Mol. Sci. 2013, 3, 296-316.

(36) Persico, M.; Granucci, G. An overview of nonadiabatic dynamics simulations methods, with focus on the direct approach versus the fitting of potential energy surfaces. Theor. Chem. Acc. 2014, 133, 1526.

(37) Crespo-Otero, R.; Barbatti, M. Recent Advances and Perspectives on Nonadiabatic Mixed Quantum-Classical Dynamics. Chem. Rev. 2018, 118, 7026-7068.

(38) Gianturco, F. A.; Jain, A. The theory of electron scattering from polyatomic molecules. Phys. Rep. 1986, 143, 347-425.

(39) Tennyson, J. Electron-molecule collision calculations using the R-matrix method. Phys. Rep. 2010, 491, 29-76.

(40) da Costa, R. F.; Varella, M. T. d. N.; Bettega, M. H. F.; Lima, M. A. P. Recent advances in the application of the Schwinger multichannel method with pseudopotentials to electron-molecule collisions. Eur. Phys. J. D 2015, 69, 159.

(41) Jolicard, G.; Austin, E. J. Optical potential stabilisation method for predicting resonance levels. Chem. Phys. Lett. 1985, 121, 106-110.

(42) Riss, U. V.; Meyer, H. D. Calculation of resonance energies and widths using the complex absorbing potential method. J. Phys. B: At. Mol. Opt. Phys. 1993, 26, 4503-4535.

(43) Hazi, A. U.; Taylor, H. S. Stabilization Method of Calculating Resonance Energies: Model Problem. Phys. Rev. A 1970, 1, 1109-1120.

(44) Mandelshtam, V. A.; Ravuri, T. R.; Taylor, H. S. The stabilization theory of scattering. J. Chem. Phys. 1994, 101, 8792-8799. 
(45) González-Ramírez, I.; Segarra-Martí, J.; Serrano-Andrés, L.; Merchán, M.; Rubio, M.; Roca-Sanjuán, D. On the $\mathrm{N}_{1}-\mathrm{H}$ and $\mathrm{N}_{3}-\mathrm{H}$ Bond Dissociation in Uracil by Low Energy Electrons: A CASSCF/CASPT2 Study. J. Chem. Theory Comput. 2012, 8, 2769-2776.

(46) Pou-Amérigo, R.; Merchán, M.; Ortí, E. Theoretical study of the electronic spectrum of p-benzoquinone. J. Chem. Phys. 1999, 110, 9536.

(47) Davis, D.; Vysotskiy, V. P.; Sajeev, Y.; Cederbaum, L. S. A one-step four-bond-breaking reaction catalyzed by an electron. Angew. Chem. Int. Ed. Engl. 2012, 51, 8003-7.

(48) Gertitschke, P. L.; Domcke, W. Time-dependent wave-packet description of dissociative electron attachment. Phys. Rev. A 1993, 4\%, 1031-1044.

(49) Lehr, L.; Miller, W. H. A classical approach to dissociative electron attachment DA: application to temperature effects in the DA cross section of $\mathrm{CF}_{3} \mathrm{Cl}$. Chem. Phys. Lett. 1996, 250, 515-522.

(50) Lehr, L.; Manz, J.; Miller, W. H. A classical approach to resonant low-energy electron scattering off molecules: application to the $a_{1}$-shape resonance of $\mathrm{CF}_{3} \mathrm{Cl}$. Chem. Phys. 1997, 214, 301-312.

(51) Pattengill, M. Classical trajectory study of three-body direct photofragmentation of $\mathrm{Cd}\left(\mathrm{CH}_{3}\right)_{2}$. Comparison of sampling methods. Chem. Phys. 1983, 75, 59-66.

(52) Pattengill, M. A classical study of the effects of parent molecule vibrational excitation prior to triatomic direct photofragmentation. Wigner versus classical weighting of trajectory initial conditions. Chem. Phys. Lett. 1984, 105, 651-654.

(53) Barbatti, M.; Sen, K. Effects of different initial condition samplings on photodynamics and spectrum of pyrrole. Int. J. Quantum Chem. 2016, 116, 762-771.

(54) Cederbaum, L. S.; Domcke, W. Local against non-local complex potential in resonant electron-molecule scattering. J. Phys. B: At. Mol. Phys. 1981, 14, 4665-4690. 
(55) Crespo-Otero, R.; Barbatti, M. Spectrum simulation and decomposition with nuclear ensemble: formal derivation and application to benzene, furan and 2-phenylfuran. Theor. Chem. Acc. 2012, 131, 1237.

(56) O'Malley, T. F. Theory of Dissociative Attachment. Phys. Rev. 1966, 150, 14-29.

(57) Sugioka, Y.; Takayanagi, T. A practical approach to temperature effects in dissociative electron attachment cross sections using local complex potential theory. Chem. Phys. 2012, 405, 189-196.

(58) Wigner, E. On the Behavior of Cross Sections Near Thresholds. Phys. Rev. 1948, 73, $1002-1009$.

(59) Akima, H. A method of univariate interpolation that has the accuracy of a third-degree polynomial. ACM Trans. Math. Softw. 1991, 17, 341-366.

(60) Tully, J. C. Molecular dynamics with electronic transitions. J. Chem. Phys. 1990, 93, 1061.

(61) Feit, M.; Fleck, J.; Steiger, A. Solution of the Schrödinger equation by a spectral method. J. Comput. Phys. 1982, 47, 412-433.

(62) Kosloff, D.; Kosloff, R. A fourier method solution for the time dependent Schrödinger equation as a tool in molecular dynamics. J. Comput. Phys. 1983, 52, 35-53.

(63) Wigner, E. On the quantum correction for thermodynamic equilibrium. Phys. Rev. 1932, 40, 749-759.

(64) Sun, L.; Hase, W. L. Comparisons of classical and Wigner sampling of transition state energy levels for quasiclassical trajectory chemical dynamics simulations. J. Chem. Phys. 2010, 133, 044313.

(65) Kossoski, F.; Barbatti, M. Nuclear Ensemble Approach with Importance Sampling. J. Chem. Theory Comput. 2018, 14, 3173-3183. 
(66) Barbatti, M.; Ruckenbauer, M.; Plasser, F.; Pittner, J.; Granucci, G.; Persico, M.; Lischka, H. Newton-X: a surface-hopping program for nonadiabatic molecular dynamics. Wiley Interdiscip. Rev. Comput. Mol. Sci. 2014, 4, 26-33.

(67) Barbatti, M.; Granucci, G.; Ruckenbauer, M.; Plasser, F.; Crespo-Otero, R.; Pittner, J.; Persico, M.; Lischka, H., NEWTON - X: A package for Newtonian dynamics close to the crossing seam. Version 2. 2016; http://www.newtonx.org, accessed May 1, 2019.

(68) Lischka, H.; Shepard, R.; Pitzer, R. M.; Shavitt, I.; Dallos, M.; Müller, T.; Szalay, P. G.; Seth, M.; Kedziora, G. S.; Yabushita, S.; Zhang, Z. High-level multireference methods in the quantum-chemistry program system COLUMBUS: Analytic MR-CISD and MRAQCC gradients and MR-AQCC-LRT for excited states, GUGA spin-orbit CI and parallel CI density. Phys. Chem. Chem. Phys. 2001, 3, 664-673.

(69) Lischka, H.; Müller, T.; Szalay, P. G.; Shavitt, I.; Pitzer, R. M.; Shepard, R. Columbusa program system for advanced multireference theory calculations. Wiley Interdiscip. Rev. Comput. Mol. Sci. 2011, 1, 191-199.

(70) Szalay, P. G.; Müller, T.; Gidofalvi, G.; Lischka, H.; Shepard, R. Multiconfiguration selfconsistent field and multireference configuration interaction methods and applications. Chem. Rev. 2012, 112, 108-81.

(71) Dunning Jr., T. H. Gaussian Basis Sets for Use in Correlated Molecular Calculations. I. The Atoms Boron Through Neon and Hydrogen. J. Chem. Phys. 1989, 90, 1007-1023.

(72) Bazante, A. P.; Davidson, E. R.; Bartlett, R. J. The benzene radical anion: A computationally demanding prototype for aromatic anions. J. Chem. Phys. 2015, 142, 204304.

(73) Langhoff, S. R.; Davidson, E. R. Configuration interaction calculations on the nitrogen molecule. Int. J. Quantum Chem. 1974, 8, 61-72. 
(74) Takatsuka, K.; McKoy, V. Theory of electronically inelastic scattering of electrons by molecules. Phys. Rev. A 1984, 30, 1734-1740.

(75) Lima, M. A. P.; Gibson, T. L.; Takatsuka, K.; McKoy, V. Multichannel Schwinger variational cross sections for electron-impact excitation of the $\mathrm{b}^{3} \Sigma_{u}^{+}$state in $\mathrm{H}_{2}$. Phys. Rev. A 1984, 30, 1741-1746.

(76) Bettega, M. H. F.; Ferreira, L. G.; Lima, M. A. P. Transferability of local-density normconserving pseudopotentials to electron-molecule-collision calculations. Phys. Rev. A 1993, 47, 1111-1118.

(77) dos Santos, J. S.; da Costa, R. F.; Varella, M. T. d. N. Low-energy electron collisions with glycine. J. Chem. Phys. 2012, 136, 084307.

(78) Kossoski, F.; Bettega, M. H. F.; Varella, M. T. d. N. Shape resonance spectra of uracil, 5-fluorouracil, and 5-chlorouracil. J. Chem. Phys. 2014, 140, 024317.

(79) Bettega, M. H. F.; Natalense, A. P. P.; Lima, M. A. P.; Ferreira, L. G. Note on the generation of Gaussian bases for pseudopotential calculations. Int. J. Quantum Chem. 1996, 60, 821-824.

(80) Skurski, P.; Gutowski, M.; Simons, J. How to choose a one-electron basis set to reliably describe a dipole-bound anion. Int. J. Quantum Chem. 2000, 80, 1024-1038.

(81) Schmidt, M. W.; Baldridge, K. K.; Boatz, J. A.; Elbert, S. T.; Gordon, M. S.; Jensen, J. H.; Koseki, S.; Matsunaga, N.; Nguyen, K. A.; Su, S.; Windus, T. L.; Dupuis, M.; Montgomery, J. A. General Atomic and Molecular Electronic Structure System. J. Comput. Chem. 1993, 14, 1347-1363.

(82) Bauschlicher, C. W. The construction of modified virtual orbitals (MVO's) which are suited for configuration interaction calculations. J. Chem. Phys. 1980, 72, 880. 
(83) Winstead, C.; McKoy, V.; Bettega, M. H. F. Elastic electron scattering by ethylene, $\mathrm{C}_{2} \mathrm{H}_{4}$. Phys. Rev. A 2005, 72, 042721.

(84) Kossoski, F.; Bettega, M. H. F. Low-energy electron scattering from the aza-derivatives of pyrrole, furan, and thiophene. J. Chem. Phys. 2013, 138, 234311.

(85) Kossoski, F.; Kopyra, J.; Varella, M. T. d. N. Anion states and fragmentation of 2chloroadenine upon low-energy electron collisions. Phys. Chem. Chem. Phys. 2015, 17, 28958-28965.

(86) Ferrer, F. J.; Cerezo, J.; Stendardo, E.; Improta, R.; Santoro, F. Insights for an accurate comparison of computational data to experimental absorption and emission spectra: Beyond the vertical transition approximation. J. Chem. Theory Comput. 2013, 9, 20722082.

(87) Aflatooni, K.; Gallup, G. A.; Burrow, P. D. Temporary Anion States of Dichloroalkanes and Selected Polychloroalkanes. J. Phys. Chem. A 2000, 104, 7359-7369.

(88) Jagau, T.-C.; Bravaya, K. B.; Krylov, A. I. Extending Quantum Chemistry of Bound States to Electronic Resonances. Annu. Rev. Phys. Chem. 2017, 68, 525-553.

(89) Jagau, T.-C. Non-iterative triple excitations in equation-of-motion coupled-cluster theory for electron attachment with applications to bound and temporary anions. J. Chem. Phys. 2018, 148, 024104.

(90) Hahndorf, I.; Illenberger, E.; Lehr, L.; Manz, J. Temperature effects of dissociative electron attachment to $\mathrm{CF}_{3} \mathrm{Cl}$. Chem. Phys. Lett. 1994, 231, 460-466.

(91) Rosa, A.; Brüning, F.; Kumar, S.; Illenberger, E. Dissociative attachment to excited $\mathrm{SF}_{6}$ : selective IR excitation versus thermal activation. Chem. Phys. Lett. 2004, 391, $361-365$. 
(92) Matejcik, S.; Ipolyi, I.; Illenberger, E. Temperature dependence of electron attachment to $\mathrm{CH}_{2} \mathrm{ClBr}$ : Competition between $\mathrm{Cl}^{-}$and $\mathrm{Br}^{-}$formation. Chem. Phys. Lett. 2003, $375,660-665$.

(93) da Costa, R. F.; Ruivo, J. C.; Kossoski, F.; Varella, M. T. d. N.; Bettega, M. H. F.; Jones, D. B.; Brunger, M. J.; Lima, M. A. P. An ab initio investigation for elastic and electronically inelastic electron scattering from para-benzoquinone. J. Chem. Phys. 2018, 149, 174308. 


\section{Graphical TOC Entry}

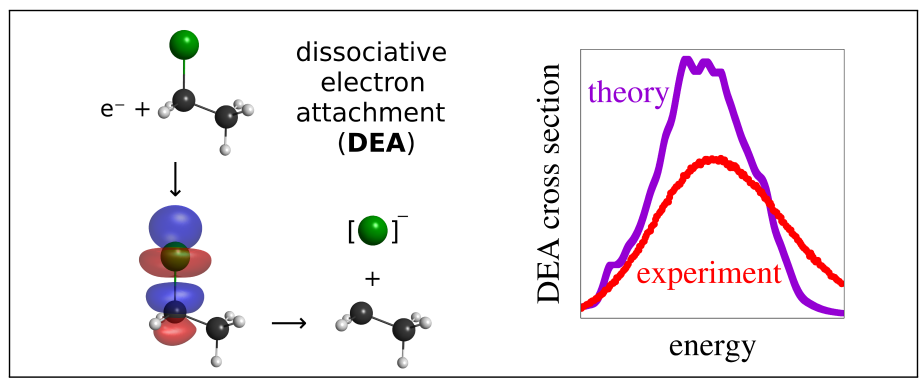

